\title{
Spectral optical monitoring of a double-peaked emission line AGN Arp 102B
}

\section{Variability of spectral lines and continuum ${ }^{\star}$}

\author{
A. I. Shapovalova ${ }^{1}$, L. Č. Popović ${ }^{2,3}$, A. N. Burenkov ${ }^{1}$, V. H. Chavushyan ${ }^{4}$, D. Ilicic 3,5 , W. Kollatschny ${ }^{6}$ \\ A. Kovačević3 ${ }^{3,5}$, N. G. Bochkarev ${ }^{7}$, J. R. Valdés ${ }^{4}$, J. Torrealba ${ }^{4}$, V. Patiño-Álvarez ${ }^{4}$, J. León-Tavares ${ }^{8,9}$, E. Benitez ${ }^{10}$, \\ L. Carrasco ${ }^{4}$, D. Dultzin ${ }^{10}$, A. Mercado ${ }^{11}$, and V. E. Zhdanova ${ }^{1}$ \\ ${ }^{1}$ Special Astrophysical Observatory of the Russian AS, Nizhnij Arkhyz, Karachaevo-Cherkesia 369167, Russia \\ e-mail: ashap@sao.ru \\ 2 Astronomical Observatory, Volgina 7, 11160 Belgrade 74, Serbia \\ 3 Isaac Newton Institute of Chile, Yugoslavia Branch, 11060 Belgrade, Serbia \\ ${ }^{4}$ Instituto Nacional de Astrofísica, Óptica y Electrónica (INAOE), Apartado Postal 51 y 216, CP 72000, Puebla, Mexico \\ 5 Department of Astronomy, Faculty of Mathematics, University of Belgrade, Studentski trg 16, 11000 Belgrade, Serbia \\ ${ }^{6}$ Institut für Astrophysik, Friedrich-Hund-Platz 1, 37077 Göttingen, Germany \\ 7 Sternberg Astronomical Institute, 119992 Moscow, Russia \\ ${ }^{8}$ Finnish Centre for Astronomy with ESO (FINCA), University of Turku, Väisäläntie 20, 21500 Piikkiö, Finland \\ 9 Aalto University Metsähovi Radio Observatory, Metsähovintie 114, 02540 Kylmälä, Finland \\ ${ }_{10}$ Instituto de Astronomía, Universidad Nacional Autónoma de Mexico, Apartado Postal 70-264, Mexico, D.F. 04510, Mexico \\ 11 Universidad Politécnica de Baja California, Av. de la Industrial 291, 21010 Mexicali, B.C., Mexico
}

Received 26 April 2013 / Accepted 10 August 2013

\section{ABSTRACT}

Context. We present results of long-term (1987-2010) optical spectral monitoring of the broad-line radio galaxy Arp 102B, a prototype of an active galactic nucleus with double-peaked broad emission lines that are commonly assumed to be emitted from an accretion disk.

Aims. To explore the structure of the broad-line region (BLR), we analyze the light-curves of the broad $\mathrm{H} \alpha$ and $\mathrm{H} \beta$ lines and the continuum flux. We aim to estimate the dimensions of the broad-line emitting regions and the mass of the central black hole.

Methods. We used the cross correlation function to find lags between the lines and continuum variations. We investigated the correlation between line and continuum fluxes in more detail and explored periodical variations of the red-to-blue line flux ratio using Lomb-Scargle periodograms.

Results. The line and continuum light-curves show several flare-like events. The fluxes in lines and in the continuum show no significant change (around 20\%) during the monitored period. We found a weak correlation between the line and continuum flux variation that may indicate that the line variation is weakly connected with the variation of the central photoionization source. In spite of this weak line-continuum correlation, we estimated a time lag for $\mathrm{H} \beta$ of about 20 days using various methods. The correlation between the $\mathrm{H} \beta$ and $\mathrm{H} \alpha$ flux variation is significantly higher than that between the lines and continuum. During the monitored period, the $\mathrm{H} \beta$ and $\mathrm{H} \alpha$ lines show double-peaked profiles, and we found an indication for a periodical oscillation in the red-to-blue flux ratio of the $\mathrm{H} \alpha$ line. The estimated mass of the central black hole is $\sim 1.1 \times 10^{8} M_{\odot}$, which agrees with the mass estimated from the $M-\sigma *$ relation.

Key words. galaxies: active - quasars: individual: Arp 102B - quasars: emission lines - line: profiles

\section{Introduction}

The broad-line emission of active galactic nuclei (AGNs) that show two peaks in the broad-line component is assumed to originate in an accretion disk (Eracleous \& Halpern 1994; Eracleous et al. 1997, 2009). These AGNs also often show a variability in the broad emission lines and continuum (see Gezari et al. 2007; Flohic \& Eracleous 2008; Shapovalova et al. 2010; Popović et al. 2011; Dietrich et al. 2012). The broad-line region (BLR) is close to the central supermassive black hole and may hold fundamental information about the formation and fueling of AGNs.

* Tables 2, 4, 6, 7 are only available at the CDS via anonymous ftp to cdsarc.u-strasbg. fr $(130.79 .128 .5)$ or via http://cdsarc.u-strasbg.fr/viz-bin/qcat?]/A+A/559/A10
Additionally, the shapes of the broad lines and their variability can give information about the BLR geometry (see Sulentic et al. 2000; Eracleous et al. 2009; Gaskell 2009).

A long-term dedicated monitoring in the optical band of some AGN nuclei has revealed a time lag in the response of the broad emission line fluxes relative to the change in the continuum flux, which depends on the size, geometry, and physical conditions of the BLR. Therefore, the search for correlations between the nuclear continuum changes and broad-line flux variations may serve as a tool for mapping the geometrical and dynamical structure of the BLR (see e.g. Peterson 1993, and reference therein).

Arp 102B is a subluminous, radio-loud LINER 1.8 galaxy at $z=0.024$ that displays double-peaked Balmer emission lines 
Table 1. Sources of spectroscopic observations.

\begin{tabular}{lcccc}
\hline \hline Observatory & Code & Tel. aperture + equipment & Aperture & Focus \\
\multicolumn{1}{c}{1} & 2 & 3 & 4 & 5 \\
\hline SAO(Russia) & L(N) & $6 \mathrm{~m}+$ Long slit & $2.0 \times 6.0$ & Nasmith \\
SAO(Russia) & L(U) & $6 \mathrm{~m}+$ UAGS & $2.0 \times 6.0$ & Prime \\
SAO(Russia) & L(Sc) & $6 \mathrm{~m}+$ Scorpio & $1.0 \times 6.07$ & Prime \\
Gullermo Haro (Mexico) & GHO & $2.1 \mathrm{~m}+\mathrm{B} \& \mathrm{C}$ & $2.5 \times 6.0$ & Cassegrain \\
San Pedro Martir (Mexico) & SPM & $2.1 \mathrm{~m}+\mathrm{B} \& \mathrm{C}$ & $2.5 \times 6.0$ & Cassegrain \\
SAO(Russia) & Z2K & $1 \mathrm{~m}+\mathrm{GAD}$ & $4.0 \times 9.45$ & Cassegrain \\
Calar Alto(Spain) & CA1 & $3.5 \mathrm{~m}+\mathrm{B} \&$ C/TWIN & $(1.5-2.1) \times 3.5$ & Cassegrain \\
Calar Alto(Spain) & CA2 & $2.2 \mathrm{~m}+\mathrm{B} \& \mathrm{C}$ & $2.0 \times 3.5$ & Cassegrain \\
\hline
\end{tabular}

Notes. Column (1): observatory. Column (2): code assigned to each combination of telescope + equipment used throughout this paper. Column (3): telescope aperture and spectrograph. Column (4): projected spectrograph entrance apertures (slit width $\times$ slit length in arcsec). Column (5): focus of the telescope.

(Stauffer et al. 1983). An accretion-disk BLR geometry has first been applied to this AGN by Chen et al. (1989) and Chen \& Halpern (1989). They used a geometrically thin, optically thick accretion disk model to fit the double-peaked Balmer lines. The accretion disk emission in the BLR of Arp 102B has been widely accepted (see Chen \& Halpern 1989; Gezari et al. 2004). Additionally, Sergeev et al. (2000) monitored the broad $\mathrm{H} \alpha$ line from 1992 to 1996 and found variations in the profile that correspond to gas rotating in a disk with inhomogeneities in the surface brightness. On the other hand, Halpern et al. (1996) found that highly ionized lines, such as Ly $\alpha$ and C IV $\lambda 1550$, do not show a disk-like profile (two peaks). These authors found that broad Mg II $\lambda 2798$ is present with nearly the same profile as the Balmer lines (peaks separated by $\sim 12000 \mathrm{~km} \mathrm{~s}^{-1}$ ) and a typical $\mathrm{Mg} \mathrm{II} / \mathrm{H} \beta$ ratio of 1 , but they found little if any C III] $\lambda 1909$ or C IV $\lambda 1550$ emission corresponding to the displaced Balmer-line peaks. Most importantly, they detected no double-peaked component in $\operatorname{Ly} \alpha$.

Newman et al. (1997) also studied the profile variability of the double-peaked $\mathrm{H} \alpha$ line in Arp 102B over 13 years and found a sinusoidal variation of the red-to-blue flux ratio from 1990 to 1994 . A similar period was found by Gezari et al. (2007). The authors modeled this variation as a transient orbiting hot spot in the accretion disk. This type of variation can also be a consequence of gravitational lensing from a massive body close to the primary black hole (Popović et al. 2001). Additionally, there are some contradictions in the disk model (see Miller \& Peterson 1990; Sulentic et al. 1990; Antonucci et al. 1996; Chen et al. 1997; Gezari et al. 2004). Moreover, recently, Fathi et al. (2011) discovered a two-armed mini-spiral structure within the inner kiloparsec resolved in the $\mathrm{H} \alpha$ line, which indicates dramatic processes happening on larger scales in the galaxy.

Studies of the variations in the continuum as well as in the broad emission line profiles and their correlations can give us information about the BLR physics (see e.g. Shapovalova et al. 2009). Here we present our investigation of a long-term optical spectral variations of Arp 102B. In this paper, we present the results of the spectral $(\mathrm{H} \alpha$ and $\mathrm{H} \beta)$ monitoring of Arp 102B during the period between 1987 and 2010, discussing the broad-line and continuum flux variability. In Paper II (Shapovalova et al. in prep.) we will give more details about the broad-line profile variability and discuss the structure and geometry of the BLR. The paper is organized as follows: in Sect. 2 we report on our observations and describe the data reduction; in Sect. 3 we describe the data analysis, and in Sect. 4 we discuss our results; finally in Sect. 5 we outline our conclusions.

\section{Observations and data reduction}

\subsection{Spectral observations}

Spectra of Arp 102B (during 142 nights) were taken with the 6-m and 1-m telescopes of the SAO RAS (Russia, 1998-2010), the INAOE 2.1-m telescope of the Guillermo Haro Observatory (GHO) at Cananea, Sonora, Mexico (1998-2007), the 2.1-m telescope of the Observatorio Astronómico Nacional at San Pedro Martir (OAN-SPM), Baja California, Mexico (2005-2007), and the 3.5-m and 2.2-m telescopes of Calar Alto observatory, Spain (1987-1994). Information on the source of the spectroscopic observations are listed in Table 1.

The SAO and Mexican spectra were obtained with longslit spectrographs equipped with CCDs. The typical observed wavelength range was $4000-7500 \AA$, the spectral resolution was $R=8-15 \AA$, and the signal-to-noise ratio ( $\mathrm{S} / \mathrm{N})$ was $20-50$ in the continuum near $\mathrm{H} \alpha$ and $\mathrm{H} \beta$. Additionally, we collected the observations taken with the Calar Alto 3.5-m and 2.2-m telescopes at ten epochs between June 1987 and September 1994. For these observations, Boller \& Chivens spectrographs were attached to the telescopes in most cases (for two epochs the TWIN spectrograph was used for the 3.5-m telescope), and different CCD detectors (RCA, GEC, Tektronix) were used. The individual spectra cover different wavelength ranges from $3630 \AA$ to $9100 \AA$, and the spectral resolution was $10-15 \AA$. The observations were taken with exposure times of 20 to $138 \mathrm{~min}$. The typical slit width was $2 . \prime 0$ projected on the sky. HeAr spectra were taken after each object exposure to enable a wavelength calibration. More information about observing conditions with the Calar Alto telescopes can be found in Kollatschny et al. (2000). From 2004 to 2007, the spectral observations with the two Mexican 2.1-m telescopes were carried out with two observational setups. We used the following configurations: 1 ) with a grating of $150 \mathrm{l} / \mathrm{mm}$ (spectral resolution of $R=15 \AA$, a resolution similar to the observations of 1998-2003); 2) with a grating of $300 \mathrm{l} / \mathrm{mm}$ (moderate spectral resolution of $R=8 \AA$ ). As a rule, spectra with $R \sim 15 \AA$ have a very good quality $(S / N>50)$, but spectra with $R \sim 8 \AA$ have more noise $(S / N \sim 20-40)$.

From 2004 to 2010 spectral observations with the 1-m Zeiss telescope of the SAO (19 nights) were carried out with the CCD $2 \mathrm{k} \times 2 \mathrm{k}$ EEV CCD42-40, which allowed us to observe the entire wavelength range (4000-8000) $\AA$ with a spectral resolution of $R=8-10 \AA$. But some of these spectra had a poor $\mathrm{S} / \mathrm{N}$ in the blue part and were not used in our analysis. Although the red part has a relatively high $\mathrm{S} / \mathrm{N}$ ratio, the spectra taken with 
the 1-m Zeiss telescope (code Z2K in Table 1) have to be treated with caution.

Spectrophotometric standard stars were observed every night. The log of the spectroscopic observations is given in Table 2. The spectrophotometric data reduction was carried out either with the software developed at the SAO RAS or with the IRAF package for the spectra obtained in Mexico and at Calar Alto. The image reduction process included bias and flatfield corrections, cosmic-ray removal, 2D wavelength linearization, sky spectrum subtraction, addition of the spectra for every night, and relative flux calibration based on observations of the standard star.

About $10 \%$ of the spectra were discarded from the analysis for several reasons (e.g. high noise, poorly corrected spectral sensitivity, poor spectral resolution (>15 $\AA$ ), etc.). Our final data set consisted of 118 blue and 90 red spectra, which were used in the analysis.

\subsection{Absolute calibration (scaling) and measurements of the spectra}

The standard flux calibration technique of spectra (i.e. comparison with stars of known spectral energy distribution) is not precise enough to study AGN variability, since even under good photometric conditions the accuracy of spectrophotometry is usually not better than $10 \%$. Therefore we used standard stars only to provide a relative flux calibration. For the absolute calibration, the fluxes of the narrow emission lines were adopted as a scaling factor of observed AGN spectra, since they are known to remain constant on timescales of tens of years (Peterson 1993).

The blue spectra were scaled using the method of van Groningen \& Wanders (1992) modified by Shapovalova et al. (2004) ${ }^{1}$. We refer to these works for details of the scaling procedure. This method allowed us to obtain a homogeneous set of spectra with the same wavelength calibration and the same [O III] $\lambda 4959+5007$ flux. Blue spectra were scaled using [O III] $\lambda 4959+5007$. Red spectra with a resolution of 8-10 do not contain these [O III] lines, but as a rule the blue spectra $(R \sim 8-10 \AA$ or $\sim 15 \AA)$ were taken in the same night. Usually, the red edge of the blue spectra and the blue edge of the red spectra overlap in an interval of $300 \AA$. Therefore, first the red spectra were rescaled using the overlapping continuum region with the blue ones. Then, the blue spectrum was scaled with the [O III $] \lambda 4959+5007$ line. In these cases, the scaling uncertainty was about $10 \%$. Then the scaling of the red spectrum was refined using the mean flux in [O I] $\lambda 6300$ determined from a low-dispersion spectrum $(R \sim 12-15 \AA)$. We have no data for [O III $] \lambda 5007+4959$ in absolute units for Arp 102B. Therefore we used the [O I] $\lambda 6300$ in absolute units from Sergeev et al. (2000) $\mathrm{F}(6300) \mathrm{abs}=(1.76 \pm 0.18) \times$ $\left(10^{-14} \mathrm{erg} \mathrm{cm}^{-2} \mathrm{~s}^{-1}\right)$ and calculated the flux for the [O III] lines to be F[O III $] 4959+5007=(4.9 \pm 0.49) \times 10^{-14} \mathrm{erg} \mathrm{cm}^{-2} \mathrm{~s}^{-1}$. For this we used the good spectra of Arp 102B that contained the $\mathrm{H} \alpha$ and $\mathrm{H} \beta$ spectral bands $(R \sim 12-15 \AA / \mathrm{px})$.

From the scaled spectra we determined the average flux in the blue continuum at the observed wavelength $\sim 5225 \AA$ (or at $\sim 5100 \AA$ in the rest frame of Arp 102B, $z=0.0241$ ) by means of the flux averaging in the bandpass of 5200-5250 $\AA$. In the red continuum the observed wavelength is $\sim 6381 \AA$ (or at $\sim 6230 \AA$ in the rest frame), averaging the flux in the wavelength range from $6356 \AA$ to $6406 \AA$. There are no strong absorption lines

\footnotetext{
1 See Appendix A in Shapovalova et al. (2004).
}

from the host galaxy in these wavelength intervals. To determine the observed fluxes of $\mathrm{H} \beta$ and $\mathrm{H} \alpha$, it is necessary to subtract the continuum contribution. To do this, a linear continuum was constructed using windows of $20 \AA$ width, located at $4700 \AA$ and $5240 \AA$ for the $\mathrm{H} \beta$ spectral band, and at $6380 \AA$ and $7000 \AA$ for that of $\mathrm{H} \alpha$. After the continuum subtraction, we defined the observed fluxes in the lines in the following wavelength intervals: (4845-5150) $\AA$ for $\mathrm{H} \beta$, and (6500-6965) $\AA$ for $\mathrm{H} \alpha$ (the interval is the same as in Sergeev et al. 2000).

\subsection{Unification of the spectral data}

To investigate the long-term spectral variability of an AGN, it is necessary to assemble a consistent, uniform data set. Because observations were carried out with four different instruments, we corrected the line and continuum fluxes for aperture effects (Peterson \& Collins 1983). As in our previous papers (Shapovalova et al. 2001, 2004, 2008, 2010, 2012), we determined a point-source correction factor $(\varphi)$ and corrected for a host galaxy contribution $(\mathrm{G})$ using the following expressions (see Peterson et al. 1995):

$F(\mathrm{H} \beta)_{\mathrm{true}}=\varphi \cdot F(\mathrm{H} \beta)_{\mathrm{obs}}$,

where $F(\mathrm{H} \beta)_{\text {obs }}$ is the observed $\mathrm{H} \beta$ flux and $F(\mathrm{H} \beta)_{\text {true }}$ is the aperture corrected $\mathrm{H} \beta$ flux. We also used

$F(\mathrm{cnt})_{\text {true }}=\varphi \cdot F(\text { cont })_{\text {obs }}-G(g)$,

where $F(\mathrm{cnt})_{\text {obs }}$ is the continuum flux at the observed wavelength and $G(g)$ is an aperture-dependent correction factor to account for the host galaxy contribution.

The GHO observing scheme (Table 1), which correspond to a projected aperture $\left(2.5^{\prime \prime} \times 6.0^{\prime \prime}\right)$ of the $2.1-\mathrm{m}$ telescope, was adopted as a standard with $\varphi=1.0$ and $G(g)=0.0$, because we collected most of the observed spectra of Arp102B with the GHO.

The correction factors $\varphi$ and $G(g)$ were determined empirically by comparing pairs of simultaneous observations from each telescope data set to that of the standard data set (as was done in AGN Watch e.g. Peterson et al. 1994, 1999, 2002). Owing to the small number of CA spectra (1987-1994), it was impossible to determine the correction factors as described above. The CA apertures for seven spectra were close to our standard (slit width $2.0^{\prime \prime}-2.1^{\prime \prime}$ ), therefore we set $\varphi=1.000$ and $G(g)=0.000$, while for only three spectra - JD 2446976 (Jun. 29, 1987), JD 2446977 (Jun. 30, 1987) and JD 2447229 (Mar. 08, 1988) the slit width was different $\left(1.5^{\prime \prime}\right)$. In these last cases we expect that the corrections are the average of the aperture 1.0" and 2.0" (Table 3). However, CA fluxes should be taken with caution. In practice, we intervals we defined as "nearly simultaneous" are typically 1-3 days long. Therefore, the variability on short timescales ( $<3$ days) is suppressed. The point-source correction factors and $G(g)$ values for different samples are listed in Table 3. Using these factors, we recalibrated the observed fluxes of $\mathrm{H} \alpha, \mathrm{H} \beta$, and the blue and red continuum to a common scale corresponding to our standard aperture $2.5^{\prime \prime} \times 6.0^{\prime \prime}$ (Table 4). We also determined the observed fluxes in the $\mathrm{H} \alpha$ and $\mathrm{H} \beta$ line segments (core and wings - the wavelength intervals are given in Table 5). Then we recalibrated the $\mathrm{H} \alpha$ and $\mathrm{H} \beta$ line segment fluxes to a common scale that corresponds to our standard aperture $2.5^{\prime \prime} \times 6.0^{\prime \prime}$ (see Tables 6, 7). The mean errors (uncertainties) in the observed continuum fluxes and in the observed fluxes of the emission lines and their segments are given in Table 5. 
Table 3. Flux scale factors for optical spectra.

\begin{tabular}{lcccc}
\hline \hline Sample & Years & $\begin{array}{c}\text { Aperture } \\
(\operatorname{arcsec})\end{array}$ & $\begin{array}{c}\text { Scale factor } \\
(\varphi)\end{array}$ & $\begin{array}{c}\text { Extended source correction } \\
\mathrm{G}(\mathrm{g})^{a}\end{array}$ \\
\hline $\mathrm{L}(\mathrm{U}, \mathrm{N})$ & $1998-2004$ & $2.0 \times 6.0$ & 1.000 & 0.000 \\
$\mathrm{~L}(\mathrm{Sc})$ & $2004-2009$ & $1.0 \times 6.0$ & 1.019 & -0.391 \\
$\mathrm{GHO}, \mathrm{SPM}$ & $1998-2007$ & $2.5 \times 6.0$ & 1.000 & 0.000 \\
Z2K & $2004-2005$ & $4.0 \times 9.45$ & 1.027 & 0.304 \\
Z2K & $2006-2010$ & $4.0 \times 9.45$ & 1.027 & 0.633 \\
CA1 & $1987-1993$ & $1.5-2.1 \times 3.5$ & $\sim 1$ & $-0.200-0.000$ \\
CA2 & $1992-1994$ & $2.0 \times 3.5$ & $\sim 1$ & 0.000 \\
\hline
\end{tabular}

Notes. ${ }^{(a)}$ In units of $10^{-15} \mathrm{erg} \mathrm{s}^{-1} \mathrm{~cm}^{-2} \AA^{-1}$.

Table 5. Error estimates for line and line-segment fluxes.

\begin{tabular}{lcccc}
\hline \hline Line & \multicolumn{2}{c}{ Spectral region } & $\sigma \pm$ e (host-galaxy corrected) & $\begin{array}{c}V_{r} \text { region } \\
{\left[\mathrm{km} \mathrm{s}^{-1}\right]}\end{array}$ \\
\hline cont 5100 & $5200-5250$ & $5077-5126$ & $3.4 \pm 2.6(9.5 \pm 6.2)$ & - \\
cont 6200 & $6356-6406$ & $6206-6255$ & $4.4 \pm 3.1(8.9 \pm 6.0)$ & - \\
$\mathrm{H} \alpha-$ total & $6500-6965$ & $6347-6801$ & $4.1 \pm 2.8(4.1 \pm 2.8)$ & $(-9875 ;+10865)$ \\
$\mathrm{H} \beta-$ total & $4845-5150$ & $4731-5028$ & $3.0 \pm 2.3(3.2 \pm 2.8)$ & $(-8074 ;+10291)$ \\
$\mathrm{H} \alpha-$ blue & $6540-6660$ & $6386-6503$ & $3.7 \pm 2.8$ & $(-8090 ;-2730)$ \\
$\mathrm{H} \alpha-$ core & $6660-6760$ & $6503-6600$ & $3.3 \pm 2.2$ & $(-2730 ;+1741)$ \\
$\mathrm{H} \alpha-$ red 1 & $6760-6860$ & $6600-6698$ & $4.5 \pm 2.2$ & $(+1739 ;+6197)$ \\
$\mathrm{H} \alpha-$ red 2 & $6920-6940$ & $6757-6776$ & $13.1 \pm 15.3$ & $(+8875 ;+9768)$ \\
$\mathrm{H} \beta-$ blue & $4854-4937$ & $4739-4820$ & $5.8 \pm 4.2$ & $(-7532 ;-2531)$ \\
$\mathrm{H} \beta-$ core & $4937-5021$ & $4820-4902$ & $4.3 \pm 3.6$ & $(-2531 ;+2531)$ \\
$\mathrm{H} \beta-$ red & $5021-5104$ & $4902-4984$ & $4.3 \pm 3.2$ & $(+2531 ;+7532)$ \\
\hline
\end{tabular}

\subsection{Host-galaxy contribution to the spectra of Arp 102B}

In the optical spectra of Arp 102B we observed a substantial starlight contribution (e.g. a strong $\mathrm{Mg}$ Ib stellar absorption line at $5176 \AA$ and a strong Na I D line at $5893 \AA$ etc.). The starlight effectively dilutes the nonstellar AGN-continuum and leads to a smaller apparent variability amplitude. The starlight also affects the profiles and fluxes of the broad Balmer lines. Therefore, we estimated the starlight contribution (i.e., host-galaxy contribution) to the fluxes of the observed continua and the $\mathrm{H} \alpha$ and $\mathrm{H} \beta$ emission lines.

For this we used spectra of Arp 102B and NGC 4339 (E0 galaxy like Arp 102B), obtained on Mar. 25, 2003 (JD 52 723.92) at the 2.1-m GHO telescope (Mexico) with an aperture of $2.5^{\prime \prime} \times 6.0^{\prime \prime}$ and a resolution of $\sim 12 \AA$ and under the same weather conditions (good transparency and seeing $\sim 2.5^{\prime \prime}$ ). We scaled the spectra of Arp 102B and NGC 4339 to $z=0$ and, changing the contribution of the galaxy NGC 4339 to Arp 102B in the region of $\mathrm{Mg} \mathrm{Ib}$ (for the blue part of the spectrum), we derived that the best-fitting ( $\mathrm{Mg}$ Ib completely removed) spectrum is obtained for $75 \pm 3 \%$ of the host-galaxy contribution to the continuum at $\sim 5100 \AA$. In the spectrum of Arp 102B the emission line at $\sim 5200 \AA$ corresponding to N II $5198 \AA$ remains, while the absorption line Na I D (at $\sim 5893 \AA$ ) is fully removed and only a weak emission line He I 5876 remains (Fig. 1). As seen from Fig. 1 (bottom), the AGN-continuum of Arp 102B has an approximately flat form and is $\sim 25 \%$ of the observed continuum at $5100 \AA$ and $6200 \AA$. We took the blue and red observed continuum fluxes for Mar. 25, 2003 (JD 52 723.92) from Table 4 and obtained the host-galaxy contribution to the observed continuum in absolute units, which is $75 \%$ of the observed continuum (see Table 8). Note that depending on the activity of the

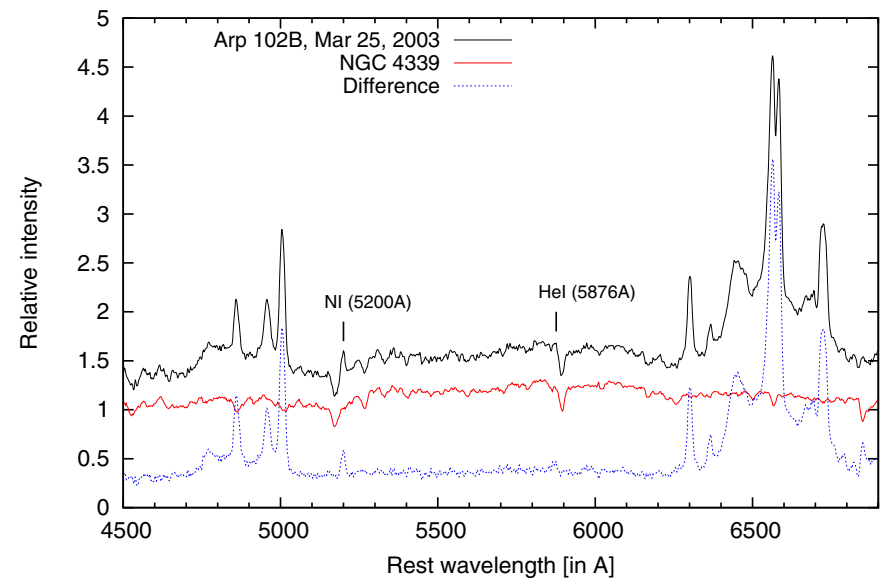

Fig. 1. Spectra of Arp 102B (top) and NGC 4339 (75\% flux of Arp 102B, middle) taken on Mar. 25, 2003, and their difference (bottom) giving the AGN continuum of Arp 102B.

AGN, the contribution of the host-galaxy continuum to the total observed continuum is between $\sim 60 \%$ and $\sim 80 \%$ (see Table 4 ).

Then we estimated the host-galaxy contribution to the $\mathrm{H} \alpha$ and $\mathrm{H} \beta$ emission line fluxes. We measured the $\mathrm{H} \alpha$ and $\mathrm{H} \beta$ line fluxes in the spectrum of Mar. 25, 2003 (JD 52 723.92) after removing the spectrum of the NGC 4339 galaxy as described above (see Fig. 1, bottom spectrum). The linear continuum in the blue (near $\mathrm{H} \beta$ ) and red (near $\mathrm{H} \alpha$ ) regions were constructed in the same way as described in Sect. 2.2. The $\mathrm{H} \alpha$ and $\mathrm{H} \beta$ line fluxes were defined in the same wavelength intervals as in Sect. 2.2. In Table 8 we list for the blue and red continua and the $\mathrm{H} \alpha$ and $\mathrm{H} \beta$ emission lines fluxes which are the observed flux, the 
Table 8. Estimate of the host-galaxy starlight contribution to the continuum (in units of $10^{-16} \mathrm{erg} \mathrm{cm}^{-2} \mathrm{~s}^{-1} \AA^{-1}$ ) and line fluxes (in units of $10^{-14} \mathrm{erg} \mathrm{cm}^{-2} \mathrm{~s}^{-1}$ ) for the spectrum taken on Mar. 25, 2003.

\begin{tabular}{lcccccc}
\hline \hline 2003 Mar. 25 & $F(5225 \AA)$ & $F(6381 \AA)$ & $F(\mathrm{H} \beta)$ & $F(\mathrm{H} \alpha)$ & $F(\mathrm{H} \beta) \mathrm{gal} / F(\mathrm{H} \beta)$ obs & $F(\mathrm{H} \alpha) \mathrm{gal} / F(\mathrm{H} \alpha) \mathrm{obs}$ \\
\hline Observed & 15.04 & 16.10 & 13.00 & 43.74 & & $4 \%$ \\
Host galaxy & 11.28 & 12.08 & 1.35 & 1.73 & $10 \%$ & $4 \%$ \\
AGN & 3.76 & 4.02 & 11.65 & 42.01 & & \\
\hline
\end{tabular}

Table 9. Contributions of the narrow lines in absolute units to the total, wings, and core flux of the $\mathrm{H} \alpha$ and $\mathrm{H} \beta$ lines (in units of $10^{-14} \mathrm{erg} \mathrm{cm}^{-2} \mathrm{~s}^{-1}$ ).

\begin{tabular}{lccccc}
\hline \hline Line fluxes & $\mathrm{H} \beta$ nar & {$[\mathrm{OIII}] 4959^{* *}$} & {$[\mathrm{OIII}] 5007$} & $(\mathrm{H} \alpha+\mathrm{NII})$ nar & {$[\mathrm{SII}] 6717,6731$} \\
\hline Mean F(nar) & $1.27 \pm 0.02$ & $1.07 \pm 0.17$ & $3.12 \pm 0.20$ & $10.86 \pm 0.34$ & $2.66 \pm 0.30$ \\
$\mathrm{~F}($ nar)/F(tot) & $9.5 \%$ & $8.0 \%$ & $24 \%$ & $24.8 \%$ & $6.1 \%$ \\
$\mathrm{~F}($ nar)/F(core) & $35.8 \%$ & & & $56.3 \%$ & \\
$\mathrm{~F}($ nar)/F(red wing) & & $32.3 \%$ & & & \\
\hline
\end{tabular}

Notes. ${ }^{(*)} F($ nar) $/ F$ (core) and $F($ nar) $/ F$ (red wing) - ratios of the narrow line flux to the mean core flux or mean red wing (in $\%) . F($ core) - mean flux for $\mathrm{H} \beta$ core and $\mathrm{H} \alpha$ core obtained from the spectra taken on Mar. 25, 2003 and Aug. 31, 2006. F (red wing) - mean flux for the red $\mathrm{H} \beta$ wing obtained from the same spectra. ${ }^{(*)}$ The ratio of the $[\mathrm{OIII}]$ lines is $5007 / 4959=2.9$ (see Dimitrijević et al. 2007).

host-galaxy contribution flux, and the AGN fluxes corrected for the host-galaxy contribution. As can be seen from Table 8, the contribution of the host-galaxy to the $\mathrm{H} \alpha$ and $\mathrm{H} \beta$ observed fluxes is $\sim 4 \%$ and $10 \%$, respectively. Then we determined the hostgalaxy corrected fluxes of all data of the blue and red continua, the $\mathrm{H} \alpha$ and $\mathrm{H} \beta$ emission lines, by subtracting from the observed flux the host-galaxy flux from Table 8 in absolute units. This is possible because the observed fluxes from Table 4 were brought (i.e., unified) to the same aperture $\left(2.5^{\prime \prime} \times 6.0^{\prime \prime}\right)$ in Sect. 2.3 and the galaxy contribution was also estimated for the same aperture. As in Sect. 2.2, we used the corrected fluxes to derive the mean errors (uncertainties) in the corrected continuum fluxes and the $\mathrm{H} \alpha$ and $\mathrm{H} \beta$ emission line fluxes by comparing fluxes in intervals of 0-3 days. The mean errors in the corrected continuum and emission line total fluxes are given in Table 5 in brackets. From Table 5 it is clear that the errors in the corrected continuum fluxes (AGN-continuum) are $\sim 2-3$ times higher than in the observed continuum fluxes, but the errors in the corrected line fluxes are close to those in the observed fluxes. The host-galaxy corrected fluxes in the blue and red continuum, the $\mathrm{H} \alpha$ and $\mathrm{H} \beta$ emission lines, and their errors are also given in Tables 4 and 5.

\subsection{Narrow emission line contribution}

To estimate the narrow-line contributions to the total (and linesegment) line fluxes, we used two spectra of Arp 102B obtained with a different spectral resolution on Mar. 25, 2003 (JD 2 452 724, $R \sim 14 \AA$ ) and Aug. 31, 2006 (JD 2453978 , $R \sim 10 \AA$ ). We estimated and subtracted the broad $\mathrm{H} \alpha$ and $\mathrm{H} \beta$ components using the spline method. The estimated contributions of the narrow $\mathrm{H} \beta$ and [OIII]4959, 5007 lines to the observed total $\mathrm{H} \beta$ flux as well as that of the narrow $\mathrm{H} \alpha+[\mathrm{NII}] 6584$ and [SII]6717, 6731 lines to the total $\mathrm{H} \alpha$ flux are given in Table 9 (in absulute units and percentage). The mean errors in the $\mathrm{H} \alpha$ and $\mathrm{H} \beta$ line fluxes are $\sim 1.5-1.8$ times higher in the corected than in the observed ones. Additionally, we estimated the narrow-line flux contributions to the $\mathrm{H} \alpha$ and $\mathrm{H} \beta$ core and $\mathrm{H} \beta$ red wing (Table 9). Note that the core and the red wing of the $\mathrm{H} \beta$ line are contaminated with only the narrow $\mathrm{H} \beta$ and [OIII]4959 lines, respectively ([OIII]5007 is beyond the red wing of $\mathrm{H} \beta$, see Table 5). The core of the $\mathrm{H} \alpha$ line is contaminated with the narrow $\mathrm{H} \alpha+[\mathrm{NII}] 6584$ lines (the [SII]6717, 6731 doublet is also beyond the red wing of the $\mathrm{H} \alpha$ line). In Table 9 we give the

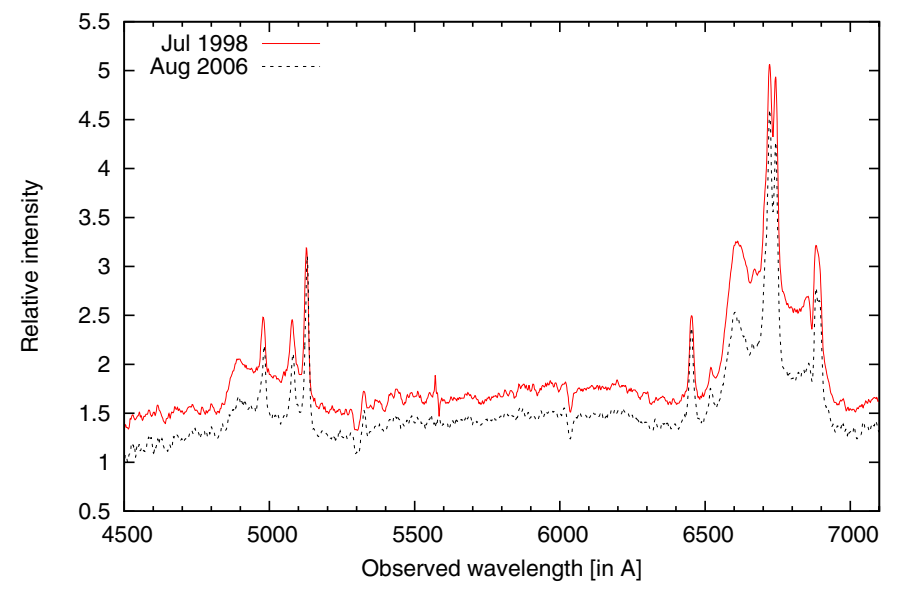

Fig. 2. Two examples of the total optical spectrum of Arp 102B, obtained when the object was in a high-activity state in July 1998 (solid line), and when it was in a low-activity state in Aug. 2006 (dashed line).

contribution (in \%) of the narrow lines to the core $\mathrm{H} \alpha$ and $\mathrm{H} \beta$, and the red wing of $\mathrm{H} \beta$ relative to the corresponding observed mean flux obtained from the spectra taken on Mar. 25, 2003 and Aug. 31, 2006.

\section{Data analysis}

We measured and analyzed variations in the continuum and lines using a total of 118 spectra covering the $\mathrm{H} \beta$ wavelength region, and 90 spectra covering the $\mathrm{H} \alpha$ line. We also considered the variability in the line segments (blue, red, and central segment) of these lines (see Tables 6 and 7), whose wavelength ranges are given in Table 5. In Fig. 2 we present two examples of the total optical spectra taken with the GHO telescope in July 1998 (when the object was in a higher state of activity) and Aug. 2006 (when the object was in a lower state of activity). In Fig. 3 we present the light-curves of the $\mathrm{H} \alpha$ and $\mathrm{H} \beta$ lines and the corresponding blue (in the rest-frame $5100 \AA$ for $\mathrm{H} \beta$ ) and red (in the rest-frame $6200 \AA$ for $\mathrm{H} \alpha$ ) continuum. The dashed lines in the first and fourth panels in Fig. 3 present the contributions of the starlight continuum of the host galaxy to the blue and red continua. The high intensity trend in the lines from 1998 is also seen in the continuum, but is absent in 1987. The variability in lines as well as 


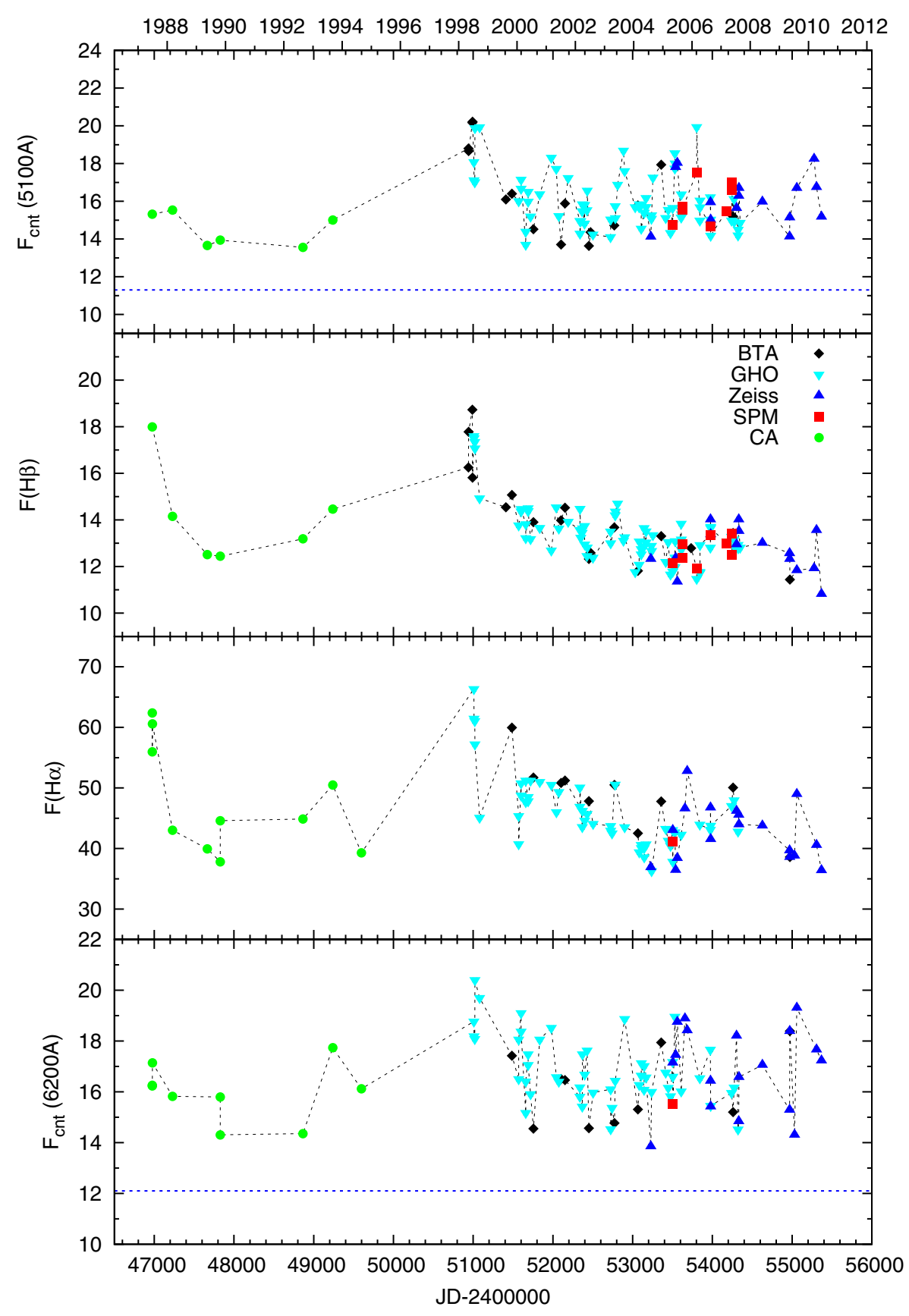

Fig. 3. Light-curves (from top to bottom) for the blue continuum flux, the $\mathrm{H} \beta$ and $\mathrm{H} \alpha$ line flux, and the red continuum flux. Observations with different telescopes are denoted with different symbols given in the middle plot. The continuum flux is plotted in units of $10^{-16} \mathrm{erg} \mathrm{cm}^{-2} \mathrm{~s}^{-1} \AA^{-1}$, and the line flux in units of $10^{-14} \mathrm{erg} \mathrm{cm}^{-2} \mathrm{~s}^{-1}$. The dashed line in the blue and red continuum light-curves mark the contribution of the host galaxy starlightcontinuum. in the continuum is moderate in the monitored period (Table 10), with several flare-like peaks.

We calculated the mean observed (obs) and corrected (cor) for the host-galaxy contribution fluxes of $\mathrm{H} \alpha, \mathrm{H} \beta$, and the blue and red continua in different periods of the monitored period and present the results in Table 12. Four observations can be made from Table 12:

a) during the monitored period the different mean observed red continuum fluxes (at $6200 \AA$ in rest frame) are always higher (at $\sim 5-7 \%$ ) than the blue continuum fluxes (at $5100 \AA$ in rest frame); this is caused by the host-galaxy contribution, because as we noted in Sect. 2.4, the corrected blue and red contina (i.e., the AGN-continuum) are almost flat;

b) in 1987 and 1998, the $\mathrm{H} \beta$ and $\mathrm{H} \alpha$ different mean observed and corrected for the host-galaxy contribution (obs and cor in Table 12) fluxes are higher for $\sim(32-35) \%(\mathrm{H} \beta)$ and $\sim(38-39) \%(\mathrm{H} \alpha)$ compared with those in the period (1988-1994) for 1987, and period (1999-2010) for 1998; this means that the variation of the mean line fluxes is independent of the host-galaxy contribution;

c) different mean red and blue continuum fluxes declined in 1988-1994 compared with the flux observed in 1987 for only $\sim(6-7) \%$, and in (1999-2010) compared with the flux observed in 1998 for (13-19)\% (Table 12). It is interesting to note that the changes in the different mean fluxes of the lines between 1987 and (1988-1994) and between 1998 and (1999-2010) are significantly larger than in the observed continuum fluxes (obs in Table 12). However, the corrected mean red and blue continuum fluxes (i.e., AGN-continuum) decreased $\sim 1.3$ times in 1988-1994 with respect to the flux observed in 1987, and $1.53-1.69$ times in 1999-2010 with respect to the flux in 1998 (see cor in Table 12). But the mean observed and corrected broad-line fluxes have almost 
A. I. Shapovalova et al.: Spectral monitoring of Arp 102B

Table 10. Parameters of the continuum and line variabilities.

\begin{tabular}{|c|c|c|c|c|c|}
\hline Feature & $N$ & $F($ mean $)$ & $\sigma(F)$ & $R(\max / \min )$ & $F($ var $)$ \\
\hline 1 & 2 & 3 & 4 & 5 & 6 \\
\hline cont 5100 & 110 & 16.04 & 1.50 & 1.48 & 0.085 \\
\hline cont 6200 & 79 & 16.79 & 1.40 & 1.47 & 0.065 \\
\hline $\mathrm{H} \alpha-$ total & 80 & 45.48 & 5.91 & 1.83 & 0.123 \\
\hline $\mathrm{H} \beta-$ total & 112 & 13.37 & 1.39 & 1.73 & 0.098 \\
\hline $\mathrm{H} \alpha$ - blue & 78 & 10.64 & 2.18 & 2.63 & 0.202 \\
\hline $\mathrm{H} \alpha$ - core & 78 & 19.73 & 2.05 & 1.64 & 0.098 \\
\hline $\mathrm{H} \alpha-\operatorname{red} 1$ & 78 & 7.73 & 1.70 & 2.99 & 0.215 \\
\hline $\mathrm{H} \alpha-\operatorname{red} 2$ & 78 & 0.38 & 0.13 & 6.94 & 0.296 \\
\hline $\mathrm{H} \beta$ - blue & 112 & 2.77 & 0.59 & 3.09 & 0.203 \\
\hline $\mathrm{H} \beta$ - core & 112 & 3.39 & 0.41 & 1.97 & 0.114 \\
\hline $\mathrm{H} \beta-$ red & 112 & 3.43 & 0.48 & 2.06 & 0.133 \\
\hline \multicolumn{6}{|l|}{+ CA data } \\
\hline cont 5100 & 115 & 15.97 & 1.51 & 1.49 & 0.086 \\
\hline cont 6200 & 88 & 16.71 & 1.39 & 1.47 & 0.064 \\
\hline $\mathrm{H} \alpha-$ total & 90 & 45.75 & 6.31 & 1.83 & 0.128 \\
\hline $\mathrm{H} \beta-$ total & 118 & 13.41 & 1.43 & 1.73 & 0.099 \\
\hline \multicolumn{6}{|l|}{ Host-galaxy-corrected data } \\
\hline cont 6200 & 88 & 4.61 & 1.39 & 4.74 & 0.287 \\
\hline $\mathrm{H} \alpha-$ total & 88 & 44.14 & 6.35 & 1.87 & 0.138 \\
\hline $\mathrm{H} \beta-$ total & 116 & 12.08 & 1.43 & 1.84 & 0.114 \\
\hline \multicolumn{6}{|l|}{ Narrow-lines-subtracted data } \\
\hline $\mathrm{H} \alpha-$ total & 88 & 30.62 & 6.35 & 2.43 & 0.203 \\
\hline $\mathrm{H} \beta$ - total & 116 & 6.62 & 1.43 & 2.97 & 0.213 \\
\hline $\mathrm{H} \alpha$ - core & 87 & 8.87 & 2.05 & 2.97 & 0.229 \\
\hline $\mathrm{H} \beta$ - core & 118 & 2.13 & 0.43 & 3.00 & 0.195 \\
\hline $\mathrm{H} \beta-$ red & 118 & 2.37 & 0.48 & 2.86 & 0.199 \\
\hline
\end{tabular}

Notes. The continuum flux is listed in units of $10^{-16} \mathrm{erg} \mathrm{cm}^{-2} \mathrm{~s}^{-1} \AA^{-1}$, and the line and line-segment fluxes in units of $10^{-14} \mathrm{erg} \mathrm{cm}^{-2} \mathrm{~s}^{-1}$. Column (1): analyzed feature of the spectrum. Column (2): total number of spectra. Column (3): mean flux. Column (4): standard deviation. Column (5): ratio of the highest to lowest flux. Column (6): variation amplitude (see text).

Table 11. Possible flares in the pure AGN-continuum (blue and red) and the corresponding changes of the $\mathrm{H} \beta$ and $\mathrm{H} \alpha$ fluxes.

\begin{tabular}{|c|c|c|c|c|c|c|c|c|c|}
\hline$N$ & UT-Date & cnt-agn5100 & Amplitude & cnt-agn6200 & Amplitude & $F(\mathrm{H} \beta)$ agn & Var & $F(\mathrm{H} \alpha)$ agn & Var \\
\hline 1 & 2 & 3 & 4 & 5 & 6 & 7 & 8 & 9 & 10 \\
\hline \multirow[t]{2}{*}{1} & 1989 Oct. 27 & & & 3.70 & $35.6 \%$ & & & 36.09 & $12 \%$ \\
\hline & 1989 Oct. 28 & & & 2.21 & & & & 42.87 & \\
\hline \multirow[t]{2}{*}{2} & 1998 Jul. 25 & 5.71 & $28 \%$ & 5.98 & $23 \%$ & 16.0 & $1.2 \%$ & 59.33 & $4.7 \%$ \\
\hline & 1998 Jul. 26 & 8.58 & & 8.30 & & 15.72 & & 55.49 & \\
\hline \multirow[t]{2}{*}{3} & 2000 Apr. 24 & 2.40 & $17.7 \%$ & 3.07 & $23.8 \%$ & 12.47 & $3.5 \%$ & 45.96 & $5.3 \%$ \\
\hline & 2000 Apr. 25 & 3.09 & & 4.31 & & 11.87 & & 49.51 & \\
\hline \multirow[t]{3}{*}{4} & 2002 Apr. 02 & 4.18 & $5.8 \%$ & & & 12.1 & $1.1 \%$ & & \\
\hline & 2002 Apr. 03 & & & 3.32 & $33.4 \%$ & & & 41.83 & $4.4 \%$ \\
\hline & 2002 Apr. 05 & 4.54 & & 5.38 & & 12.29 & & 44.51 & \\
\hline \multirow[t]{3}{*}{5} & 2003 Mar. 24 & 2.81 & $20 \%$ & & & 12.15 & $3.0 \%$ & & \\
\hline & 2003 Mar. 25 & 3.74 & & 4.0 & $34.5 \%$ & 11.65 & & 42.01 & $1.3 \%$ \\
\hline & 2003 Mar. 26 & & & 2.43 & & & & 41.23 & \\
\hline \multirow[t]{3}{*}{6} & 2004 Apr. 11 & 4.51 & $23 \%$ & 4.54 & $7.2 \%$ & 11.4 & $1.4 \%$ & 38.88 & $1.0 \%$ \\
\hline & 2004 Apr. 12 & 3.25 & & & & 11.17 & & & \\
\hline & 2004 Apr. 13 & & & 5.03 & & & & 38.36 & \\
\hline \multirow[t]{6}{*}{7} & 2006 Aug. 28 & 3.38 & $19.8 \%$ & 5.56 & $25.5 \%$ & 12.01 & $3.3 \%$ & 41.38 & $5.2 \%$ \\
\hline & 2006 Aug. 28 & 4.92 & & 4.33 & & 11.46 & & & \\
\hline & 2006 Aug. 29 & 4.65 & & & & 12.67 & & 45.02 & \\
\hline & 2006 Aug. 30 & 3.72 & & 3.32 & & 12.03 & & 39.82 & \\
\hline & 2006 Aug. 30 & 3.73 & & & & 12.35 & & & \\
\hline & 2006 Aug. 31 & 2.88 & & 3.35 & & 12.30 & & 42.06 & \\
\hline \multirow[t]{3}{*}{8} & 2009 May 17 & 2.83 & $21.4 \%$ & 3.19 & $34 \%$ & 11.22 & $1.6 \%$ & 37.95 & $1.7 \%$ \\
\hline & 2009 Мay 19 & 3.84 & & 6.29 & & 10.97 & & 36.88 & \\
\hline & 2009 May 20 & & & 6.29 & & & & 36.86 & \\
\hline
\end{tabular}

Notes. Column (1): number of the possible flare. Column (2): date. Columns (3) and (5): pure AGN blue (at $5100 \AA$ ) and red (at $6200 \AA$ ) continuum flux, corrected for the host-galaxy contribution, in units of $10^{-16} \mathrm{erg} \mathrm{cm}^{-2} \mathrm{~s}^{-1} \AA^{-1}$. Columns (4) and (6): flare amplitude in \%. Columns (7) and (9): $\mathrm{H} \beta$ and $\mathrm{H} \alpha$ fluxes corrected for the host-galaxy contribution, in units of $10^{-14} \mathrm{erg} \mathrm{cm}^{-2} \mathrm{~s}^{-1}$. Columns (8) and (10): The change in the line flux in \%. 

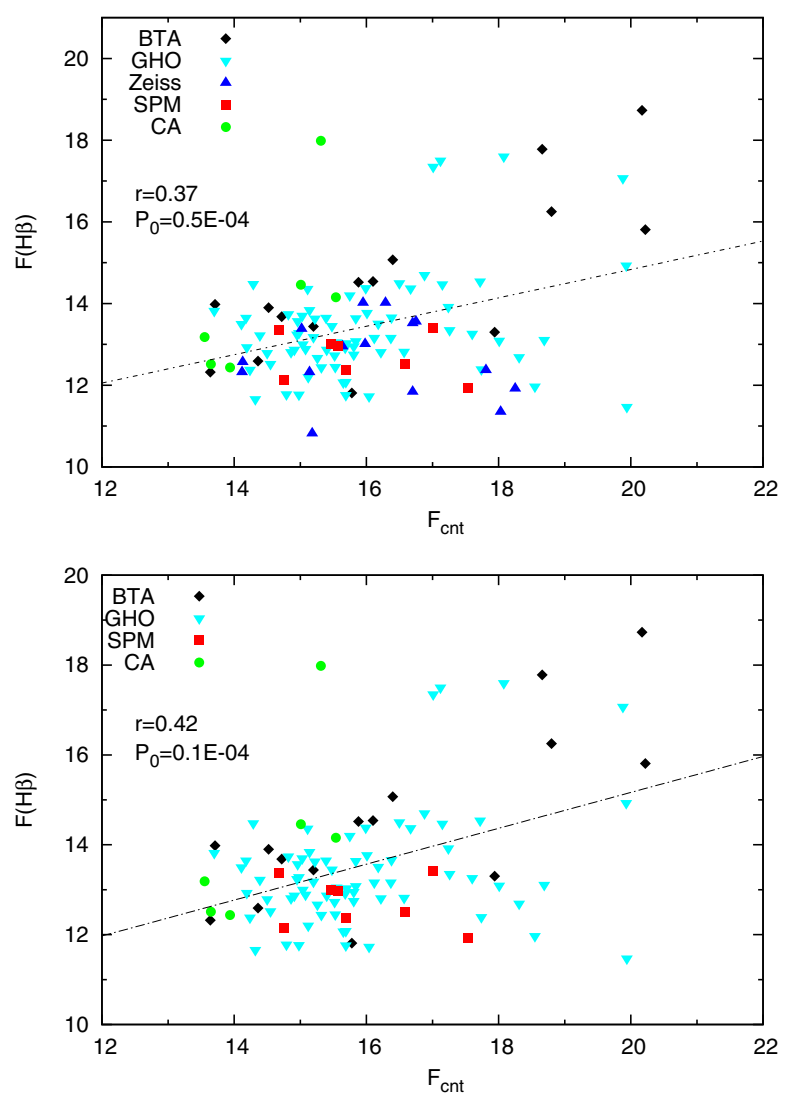

Fig. 4. $\mathrm{H} \beta$ vs. continuum flux. Upper plot: all observed data. Bottom: without the data from the Zeiss telescope. The continuum flux is plotted in units of $10^{-16} \mathrm{erg} \mathrm{cm}^{-2} \mathrm{~s}^{-1} \AA^{-1}$, the line flux in units of $10^{-14} \mathrm{erg} \mathrm{cm}^{-2} \mathrm{~s}^{-1}$. Observations with different telescopes are denoted with different symbols given in the upper left corner. The correlation coefficient and the corresponding $p$-value are also given.

the same variability amplitude in these periods $(\sim 1.35)$, that is, it does not depend on the host-galaxy contribution;

d) the mean broad $\mathrm{H} \alpha$ and $\mathrm{H} \beta$ line fluxes that were corrected for the narrow-line contributions are given in Table 12 (see: cor-line). Obviously, the change of the mean line fluxes (decrease of $\sim 1.46-1.63$ times in 1999-2010 with respect to the flux observed in 1998) is slightly smaller than in the blue-red continuum flux changes, but it is significantly higher than in the case where the narrow-line contributions are not taken into account.

\subsection{Variability of the emission lines and continuum}

To estimate the variability in different line segments, we used the method reported by O'Brien et al. (1998) and defined several parameters that characterize the variability of the continuum, total line, and line-segments fluxes (Table 10). There, $N$ is the number of spectra, $F$ denotes the mean flux over the whole observing period, $\sigma(F)$ is the standard deviation, and $R(\mathrm{max} / \mathrm{min})$ is the ratio of the highest to lowest flux in the monitored period. The parameter $F$ (var) is an inferred (uncertainty-corrected) estimate of the variation amplitude with respect to the mean flux, defined as

$F($ var $)=\left[\sqrt{\sigma(F)^{2}-e^{2}}\right] / F($ mean $)$,

where $e^{2}$ is the mean-square value of the individual measurement uncertainty for $N$ observations, i.e., $e^{2}=\frac{1}{N} \sum_{i}^{N} e(i)^{2}$ (O'Brien et al. 1998). As can be seen from Table 10, the variability indicator $F$ (var) is low ( $\sim 10-12 \%$ for the observed $\mathrm{H} \alpha$ and $\mathrm{H} \beta, \sim 9 \%$
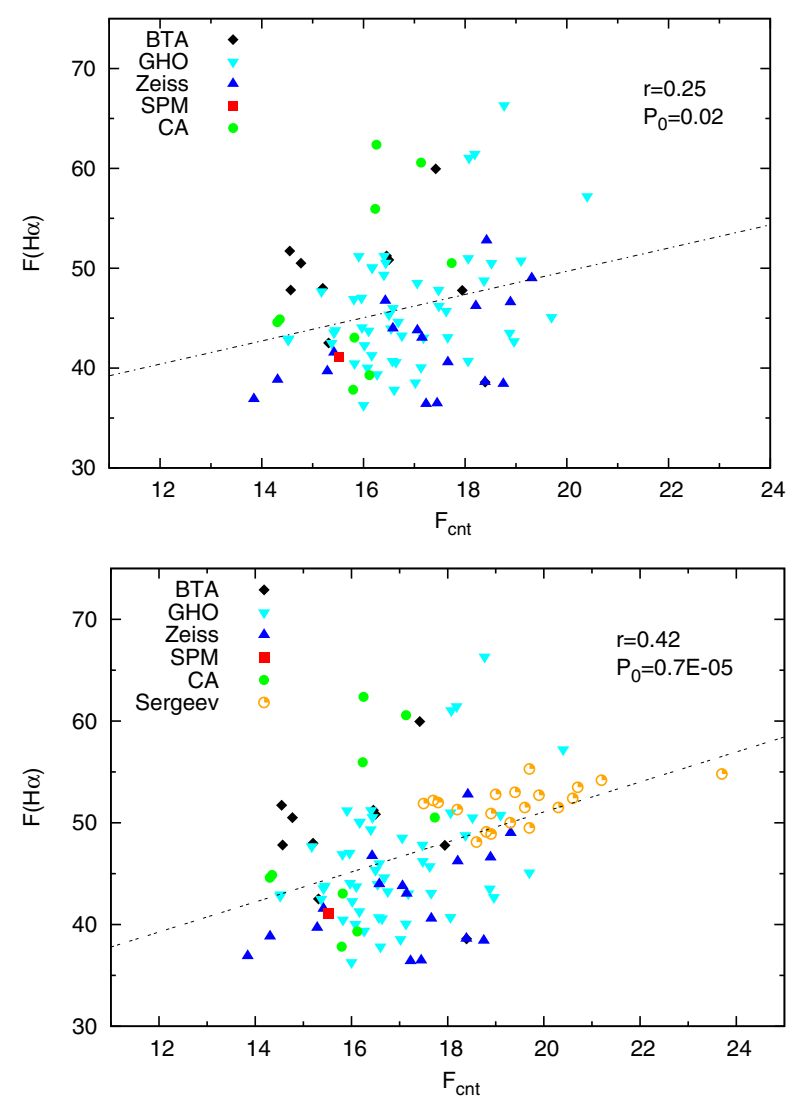

Fig. 5. Same as Fig. 4, but for $\mathrm{H} \alpha$ (upper panel). Bottom panel also includes data from Sergeev et al. (2000).

for the continuum at $5100 \AA$, and $\sim 7 \%$ for the continuum at $6200 \AA$ ). The blue wing of $\mathrm{H} \alpha$ and $\mathrm{H} \beta$ and $\mathrm{H} \alpha$-red 1 wing vary more $(\sim 20 \%)$ than the corresponding line cores $(\sim 11 \%)$ and the red wing of $\mathrm{H} \beta(\sim 13 \%)$. But the relative variation amplitude $F$ (var) of the continuum fluxes changed more much $(\sim 30 \%)$ when we removed the host-galaxy contribution (i.e., the corrected or AGN-continuum), while $F$ (var) of the $\mathrm{H} \alpha$ and $\mathrm{H} \beta$ line fluxes remained almost unchanged (host-galaxy-corrected data in Table 10). In the corrected blue and red continuum lightcurves we found some possible flare-like events with an amplitude of up to $30 \%$ that last for a few (2-3) days (see Table 11), while in the corrected $\mathrm{H} \alpha$ and $\mathrm{H} \beta$ line light-curves we observed no flare-like events at the corresponding epochs.

Note here that the narrow-line contamination of the $\mathrm{H} \alpha$ and $\mathrm{H} \beta$ broad lines and their line segments can affect the variation amplitude $F$ (var). These contaminations may cause the measured small variation in the $\mathrm{H} \alpha$ and $\mathrm{H} \beta$ core and in the $\mathrm{H} \beta$ red wing. We corrected the $\mathrm{H} \alpha$ and $\mathrm{H} \beta$ line fluxes and their segments for the narrow-line contribution (Table 10, narrow-line subtracted data) and obtained that the variability indicator $F$ (var) is $\sim 20 \%$ in the corrected $\mathrm{H} \alpha$ and $\mathrm{H} \beta$ line and line-segment fluxes.

We should also note that the part of the $\mathrm{H} \alpha$ red wings ( $\mathrm{H} \alpha$-red 2 in Tables 5-10) is very weak and its contribution to the $\mathrm{H} \alpha$ flux is negligible. We used the $\mathrm{H} \alpha$-red2 flux only to investigate variations in the red-to-blue line-segment flux ratio (see Sect. 3.2.1).

In Figs. 4 and 5 we plot the $\mathrm{H} \beta$ and $\mathrm{H} \alpha$ line fluxes as a function of the continuum. Because the observations made with the Zeiss telescope should be taken with caution, we present the line vs. continuum flux with and without data obtained with the Zeiss 

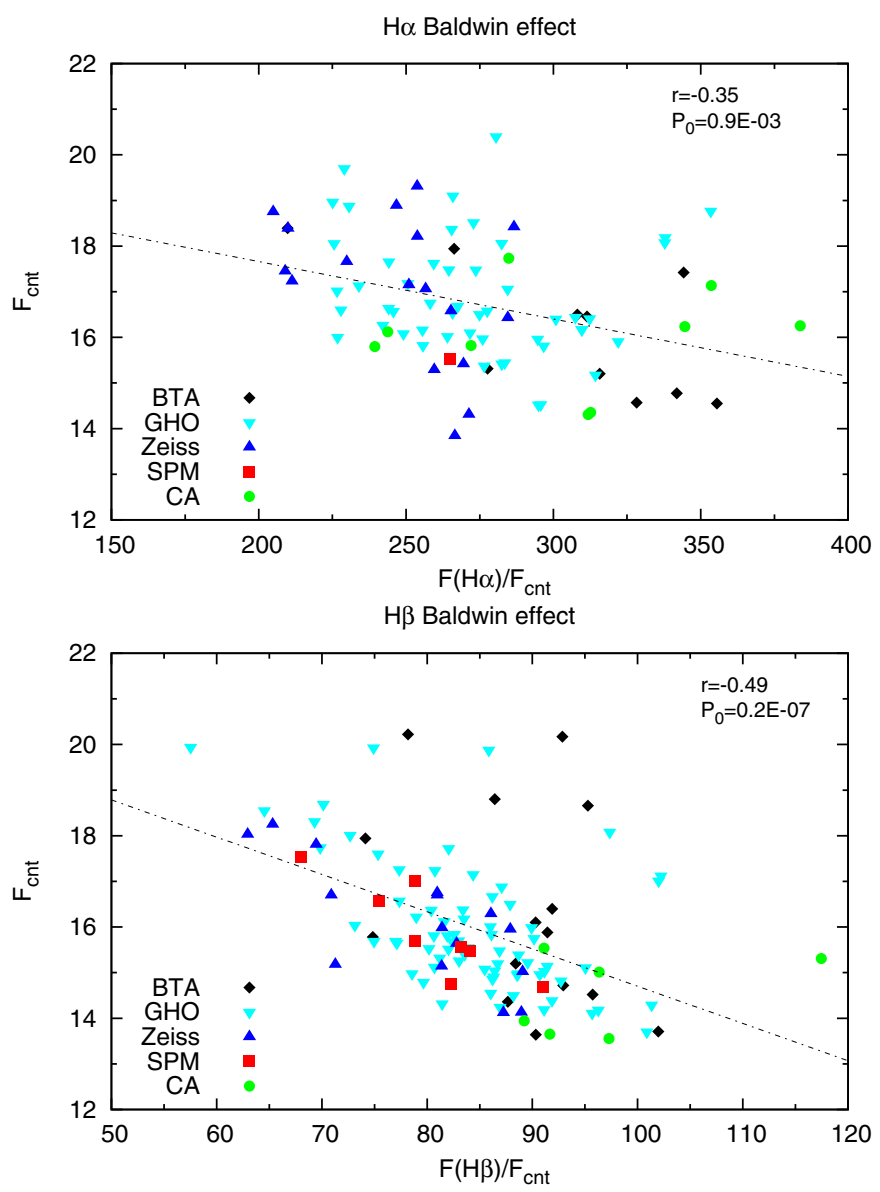

Fig. 6. Baldwin effect in the $\mathrm{H} \alpha$ (upper panel) and $\mathrm{H} \beta$ line (bottom panel). The continuum flux is plotted in units of $10^{-16} \mathrm{erg} \mathrm{cm}^{-2} \mathrm{~s}^{-1} \AA^{-1}$. Observations with different telescopes are denoted with different symbols given in the bottom left corner. The correlation coefficient and the corresponding $p$-value are also given.

telescope (see Tables 1 and 2, code Z2K). As can be seen from the figures, the line and continuum fluxes are only weakly correlated, $r=0.42$ for $\mathrm{H} \beta$ and 0.42 for $\mathrm{H} \alpha$ (without the Zeiss telescope data). This weak correlation between the line and the continuum flux also indicates that there may be present other effects in addition to the continuum central source that contribute to the photoionization.

On the other hand, in Fig. 6 we plot the intrinsic Baldwin effect, and there is a clear anticorrelation between the continuum flux and the equivalent widths of the $\mathrm{H} \beta\left(r=-0.49, P_{0}=0.6 \times\right.$ $\left.10^{-07}\right)$ and $\mathrm{H} \alpha$ lines $\left(r=-0.35, P_{0}=0.9 \times 10^{-03}\right)$, which means that there is an intrinsic Baldwin effect in the $\mathrm{H} \beta$ and $\mathrm{H} \alpha$ lines, similar to that observed in another (single peaked) AGNs (see e.g. Gilbert \& Peterson 2003).

We defined the observed fluxes in the blue and red wings and in the core of the $\mathrm{H} \alpha$ and $\mathrm{H} \beta$ lines (Tables 6, 7) in the wavelength intervals listed in Table 5. In Fig. 7 we plot the $\mathrm{H} \alpha$ and $\mathrm{H} \beta$ line-wing fluxes (blue, red) vs. line-core flux (upper panels), and red vs. blue-wing (bottom panel). The dashed line in Fig. 7 represents the best fit, while the solid line represents the expected slope for the case where the different parts of the line profile vary proportionally to each other, that is, the slope of the best fit is 1 .

As can be seen in Fig. 7, the variation correlation between the blue/red wings and central component is high, as is that between the red and blue wings $(r \sim 0.8)$. However, the slopes of the best-fit lines are not consistent with 1 , except for the $\mathrm{H} \beta$ red wing vs. core, where the best fit is very close to 1 .

Although the correlations between the $\mathrm{H} \alpha$ vs. $\mathrm{H} \beta$ total line and line-segment fluxes (Fig. 9) are very good $(r \sim 0.8)$, the best fits are far away from the slope of 1 . Moreover, for the line cores, $F(\mathrm{H} \alpha)$ core vs. $F(\mathrm{H} \beta)$ core, the correlation coefficient is lower $(r \sim 0.65)$. On the other hand, the correlation between the line segment and continuum flux is very low, almost absent, and is statistically not important for $\mathrm{H} \alpha$ (see Fig. 8). The situation is slightly better for the $\mathrm{H} \beta$ line.

\subsubsection{Cross-correlation analysis}

The light-curves shown in Fig. 3 are complex, with several peaks, and the observed fluxes show only modest variations, which is indicated by the $F$ (var) parameter in Table 10 . In spite of the weak correlation between the line and continuum fluxes, we applied the $Z$-transformed discrete correlation function method, called ZDCF (see Alexander 2013) on our data samples. We performed several calculations, using different number of data. First of all, we discarded poor Zeisstelescope spectra and also calculated the cross-correlation function (CCF) using only spectra observed with two telescopes from Mexico. The results of the cross-correlation analysis are given in Table 13

The continuum and both line-emission light-curves $\mathrm{H} \alpha$ and $\mathrm{H} \beta$ (without the poor Zeiss-telescope spectra) varies similarly. As can be seen from Table 13, the lags for $\mathrm{H} \beta$ for a different number of points range from 20 to 29 days, and for $\mathrm{H} \alpha$ from 17 to 29 days. The cross-correlation coefficients are small, but the ZDCF coefficient is higher for $\mathrm{H} \beta$.

Additionally, we applied the method of $\mathrm{Zu}$ et al. (2011) to estimate the lag of the $\mathrm{H} \beta$ line (Fig. 10). We also applied this method on the $\mathrm{H} \alpha$ line, but it did not produce valid results. In these calculations, the first step is to build a continuum model to determine the $\mathrm{Zu}$ model parameters of the continuum light-curve. The continuum light-curve is generated from the model with a timescale of 100 days and a variability amplitude of $\sigma=2$. The posterior distribution of the two parameters of the continuum variability $\left(\tau_{\mathrm{d}}, \sigma\right)$ are calculated from the $\mathrm{Zu}$ model using 40000 MCMC (Markov chain Monte Carlo) method burnin iterations. To measure the lag between the continuum and the $\mathrm{H} \beta$ light-curve, the $\mathrm{Zu}$ model then interpolates the continuum light-curve based on the posteriors $\left(\tau_{\mathrm{d}}, \sigma\right)$ derived, and then shifts, smooths, and scales continuum light-curve to compare to the observed $\mathrm{H} \beta$ light-curve. After repeating this 10000 times in an MCMC run, we derived the posterior distribution of the lag, the tophat width $\mathrm{w}$, and the scale factor $\mathrm{s}$ of the emission line, along with updated posteriors for the timescale $\tau_{\mathrm{d}}$ and the amplitude $\sigma$ of the continuum. The model gives for the lag between the blue continuum and $\mathrm{H} \beta$ line $36.95_{+19.72}^{-47.53}$ days and for the red continuum and $\mathrm{H} \alpha$ line the lag is $22.86_{+16.84}^{-30.20}$ days, which is approximately within $3 \sigma$ distance from the lag values between the continuum and emission lines obtained by the classical methods given in Table 13. To determine the best-fitting parameters from the last MCMC run, we compare in Fig. 10 the best-fitting and the observed light-curves. Clearly, the observation between MJD 46975 and 50991 are closer to the best-fitting light-curve for $\mathrm{H} \alpha$ than for the $\mathrm{H} \beta$ line.

Finally, we applied the interpolation cross-correlation function method (ICCF) method (Bischoff \& Kollatschny 1999) to cross-correlate the flux of the continuum with the $\mathrm{H} \beta$ and $\mathrm{H} \alpha$ flux. In this case, the error-bars in the lags were large 

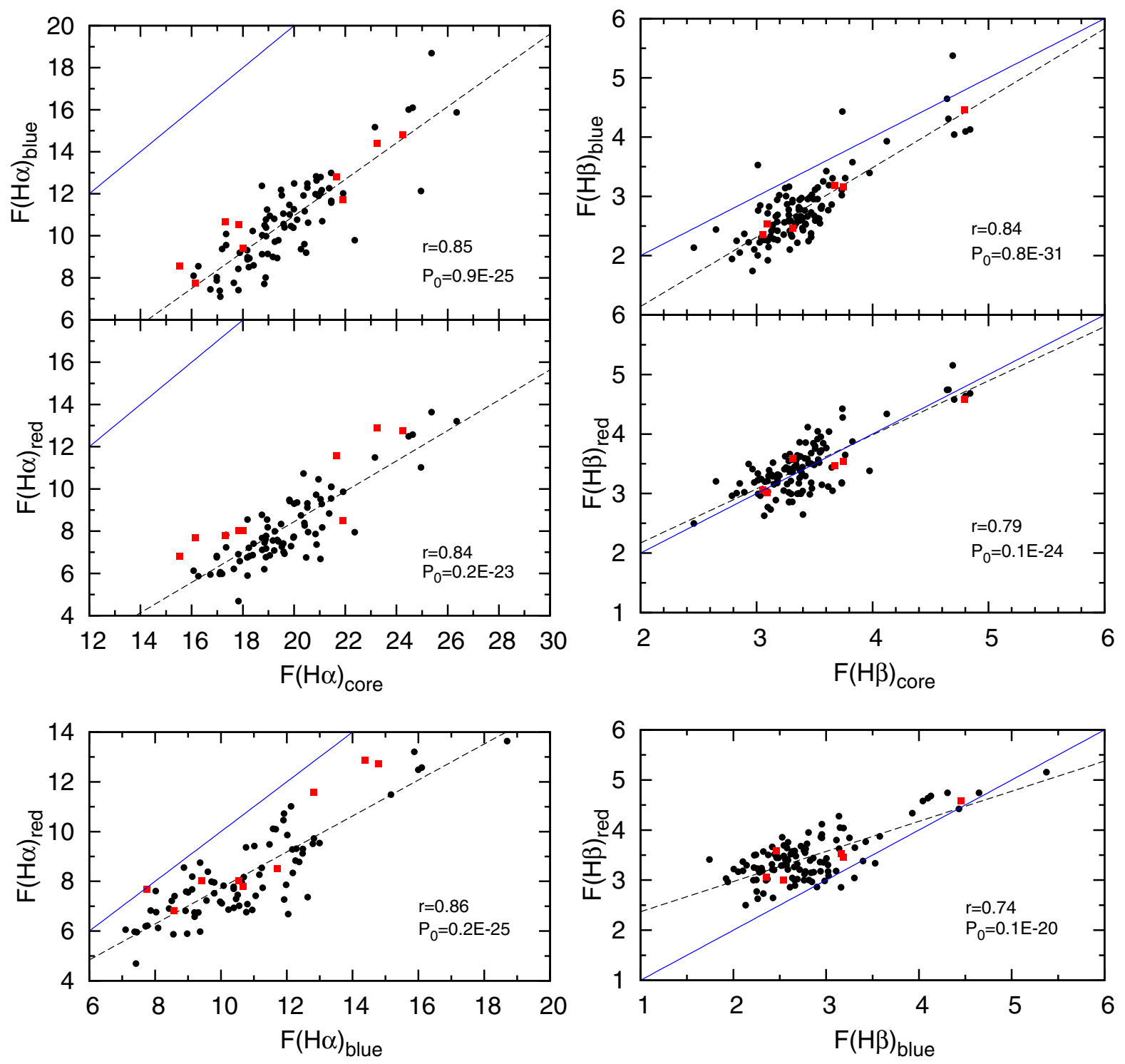

Fig. 7. $\mathrm{H} \alpha$ and $\mathrm{H} \beta$ line-wing fluxes (blue, red) vs. line-core flux (upper panels), and red vs. blue-wing (bottom panel). The line-segment fluxes are plotted in units of $10^{-14} \mathrm{erg} \mathrm{cm}^{-2} \mathrm{~s}^{-1}$. The correlation coefficient and the corresponding $p$-value are given in the bottom right corner. The CA-data are denoted with squares. The dashed line gives the linear best fit of the data, while the solid line marks the linear function with the slope equal to 1 .

as well and there is an indication for a lag of 20 days, which agrees with the previous two methods. Therefore, we assume a time lag for $\mathrm{H} \beta$ of 20 days for the black hole mass estimation.

\subsection{Changes in the broad-line profiles}

We discuss and model the line shape variability in Paper II, here we provide some characteristics of the line profile and peak variations, because Arp 102B is a prototype of double-peaked emitters.

During the monitored period, the broad $\mathrm{H} \beta$ and $\mathrm{H} \alpha$ lines showed double-peaked profiles. In Fig. 11 we present the mean profile of the $\mathrm{H} \beta$ and $\mathrm{H} \alpha$ lines and their rms profile (top panels) and the corresponding ratio of the rms-to-mean profile (bottom panels). The FWHM of the mean and rms profiles are: $\mathrm{H} \alpha$ mean $14320 \mathrm{~km} \mathrm{~s}^{-1}$ and rms $14450 \mathrm{~km} \mathrm{~s}^{-1}$, and $\mathrm{H} \beta$ mean $15900 \mathrm{~km} \mathrm{~s}^{-1}\left(15840 \mathrm{~km} \mathrm{~s}^{-1}\right.$ ) and rms 14870 (or 16080 if not corrected for the underlying continuum). The distance between the two peaks is about $11000 \mathrm{~km} \mathrm{~s}^{-1}$, the blue peak is located at about $-5000 \mathrm{~km} \mathrm{~s}^{-1}$ and the red peak at about $6000 \mathrm{~km} \mathrm{~s}^{-1}$ from the line center. These large distances between the peaks indicate a fast rotating disk that is probably close to the black hole. As can be seen from Fig. 11, the changes in the line profile also show two peaked rms, which indicates that the changes in the broad-line profile are in both the red and blue peaks in both lines, but the changes in the blue wing are significantly stronger, than in the red one. One central peak in the rms may be caused by a central component (see e.g. Popović et al. 2004; Bon et al. 2006, 2009).

\subsubsection{Red-to-blue peak ratio}

Newman et al. (1997) and Sergeev et al. (2000) reported that the variation in the red-to-blue flux ratio of $\mathrm{H} \alpha$ that a periodical characteristic. The observed wavelength intervals of $\mathrm{H} \alpha$ are defined in such a way that the red wing excludes the narrow forbidden lines of [N II] $\lambda 6584$ and [S II] $\lambda \lambda 6717,6731(\mathrm{H} \alpha-$ red 1 and $\mathrm{H} \alpha-\operatorname{red} 2$ from Table 5). The blue and red wavelength 
Table 12. Mean observed (obs), host-galaxy-corrected (cor) and narrow-line-subtracted (cor-line) fluxes and standard deviations of $\mathrm{H} \alpha$, $\mathrm{H} \beta$, and the blue and red continuum in different monitored periods.

\begin{tabular}{|c|c|c|c|c|c|c|}
\hline $\begin{array}{c}\text { UT-date } \\
1\end{array}$ & & $\begin{array}{c}\text { JD period } \\
2\end{array}$ & $\begin{array}{c}F_{\text {cnt }}(5100) \pm \sigma \\
3\end{array}$ & $\underset{4}{F(\mathrm{H} \beta)} \pm \sigma$ & $\begin{array}{c}F(\mathrm{H} \alpha) \pm \sigma \\
5\end{array}$ & $\begin{array}{c}F_{\text {cnt }}(6200) \pm \sigma \\
6\end{array}$ \\
\hline \multirow{3}{*}{1987} & obs & 46976 & $15.31^{*}$ & $17.99^{*}$ & $59.64 \pm 3.31(5.6 \%)$ & $16.54 \pm 0.51(3.1 \%)$ \\
\hline & cor & & $4.01^{*}$ & $16.64^{*}$ & $57.91 \pm 3.32(5.7 \%)$ & $4.44 \pm 0.51(11.5 \%)$ \\
\hline & cor-line & & - & $11.18^{*}$ & $44.39 \pm 3.32(7.5 \%)$ & - \\
\hline \multirow[t]{3}{*}{ 1988-1994 } & obs & 48413 & $14.34 \pm 0.89(6.2 \%)$ & $13.35 \pm 0.93(6.9 \%)$ & $42.87 \pm 4.33(10.1 \%)$ & $15.69 \pm 1.27(8.1 \%)$ \\
\hline & cor & & $3.04 \pm 0.89(29.2 \%)$ & $12.00 \pm 0.93(7.7 \%)$ & $41.63 \pm 4.53(10.9 \%)$ & $3.59 \pm 1.27(35.4 \%)$ \\
\hline & cor-line & & - & $7.31 \pm 2.07(28.3 \%)$ & $28.11 \pm 4.53(16.1 \%)$ & - \\
\hline \multirow[t]{3}{*}{ 1987/(1988-1994) } & obs & & 1.07 & 1.35 & 1.39 & 1.05 \\
\hline & cor & & 1.32 & 1.39 & 1.39 & 1.24 \\
\hline & cor-line & & - & 1.53 & 1.58 & - \\
\hline \multirow[t]{3}{*}{1998} & obs & $50940-51021$ & $18.74 \pm 1.29(6.9 \%)$ & $17.26 \pm 0.91(5.3 \%)$ & $61.52 \pm 3.73(6.1 \%)$ & $18.86 \pm 1.07(5.7 \%)$ \\
\hline & cor & & $7.57 \pm 1.27(16.7 \%)$ & $15.65 \pm 1.15(7.4 \%)$ & $56.51 \pm 8.02(14.2 \%)$ & $6.93 \pm 1.00(14.4 \%)$ \\
\hline & cor-line & & - & $10.19 \pm 1.15(11.3 \%)$ & $42.99 \pm 8.02(18.7 \%)$ & - \\
\hline \multirow[t]{3}{*}{ 1999-2010 } & obs & $51410-55367$ & $15.79 \pm 1.24(7.8 \%)$ & $13.05 \pm 0.86(6.6 \%)$ & $44.63 \pm 4.70(10.5 \%)$ & $16.64 \pm 1.29(7.8 \%)$ \\
\hline & cor & & $4.49 \pm 1.24(27.5 \%)$ & $11.72 \pm 0.85(7.3 \%)$ & $42.95 \pm 4.76(11.1 \%)$ & $4.54 \pm 1.29(28.5 \%)$ \\
\hline & cor-line & & - & $6.26 \pm 0.85(13.6 \%)$ & $29.43 \pm 4.76(16.2 \%)$ & - \\
\hline \multirow[t]{3}{*}{ 1998/(1999-2010) } & obs & & 1.19 & 1.32 & 1.38 & 1.13 \\
\hline & cor & & 1.69 & 1.34 & 1.32 & 1.53 \\
\hline & cor-line & & - & 1.63 & 1.46 & - \\
\hline
\end{tabular}

Notes. The continuum flux is listed in units of $10^{-16} \mathrm{erg} \mathrm{cm}^{-2} \mathrm{~s}^{-1} \AA^{-1}$, the line flux in units of $10^{-14} \mathrm{erg} \mathrm{cm}^{-2} \mathrm{~s}^{-1}$. - Column (1): observed period Column (2): Julian date period in units of $2400000+$. Columns (3-6): mean flux and standard deviations of blue continuum, $\mathrm{H} \beta, \mathrm{H} \alpha$, and red continuum, the error in percentages is given in brackets. The middle and last row give the ratio of the mean fluxes in 1987 and in 1988-1994, and in 1998 and in 1999-2010, respectively. ${ }^{(*)}$ For year 1987 it was not possible to derive a standard deviation for the blue continuum and $\mathrm{H} \beta$ line flux.

intervals (see Table 5) correspond to intervals from Newman et al. (1997) for $\mathrm{H} \alpha$. We measured the line-segment flux ratio for $\mathrm{H} \alpha$ and $\mathrm{H} \beta$ (Tables 6, 7) and applied a Lomb-Scargle periodogram (Lomb 1976; Scargle 1982) to find possible periodical variations in this ratio.

Figure 12 gives the Lomb-Scargle periodograms of the ratio of the red-to-blue line-segment fluxes $(R=F($ red $) / F$ (blue) $)$ of the $\mathrm{H} \alpha$ and $\mathrm{H} \beta$ lines. As can be seen in Fig. 12, there are three peaks, one of which is clearly distinguished and higher than $0.01(99 \%)$ of the false-alarm probability (FAP) ${ }^{2}$. This peak (denoted as P-peak) at an angular frequency of $\sim 0.017$ for both lines corresponds to a period of $\sim 370$ days. The angular frequency of 0.00795 corresponding to the period of $\sim 790$ days found by Newman et al. (1997) and Gezari et al. (2007) (denoted with $N$ ) seems to be located between two other peaks of lower significance (denoted as 1- and 2-peak in Fig. 12): the first peak is at an angular frequency of $\sim 0.00968$ for $\mathrm{H} \alpha(0.0094$ for $\mathrm{H} \beta$ ) corresponding to a period of $\sim 650$ days (670 days), and the second peak is at an angular frequency of $\sim 0.0074(0.0077)$ corresponding to a period of $\sim 850$ days ( 815 days).

\section{Discussion}

Before discussing our results, we point out that the host galaxy of Arp 102B is an E-galaxy with strong stellar absorption lines ( $\mathrm{Mg} \mathrm{Ib}, \mathrm{Na}$ ID, CaII H, K, etc.) typical for this type of galaxies. The host-galaxy continuum can strongly affect the observed spectra, therefore we determined the host-galaxy contribution to the blue and red fluxes of the observed continuum as well as the

\footnotetext{
2 The false-alarm probability (FPA) describes the probability that at least one out of $\mathrm{M}$ independent power values in a prescribed search band of a power spectrum computed from a white-noise time series is expected to be as high as or higher than a given value. Note that the low FAP values indicate a high degree of significance in the associated periodic signal.
}

Table 13. Sampling characteristics and cross-correlation analysis of $\mathrm{H} \beta$.

\begin{tabular}{lccc}
\hline \hline Light curve & $N$ & Lag ZDCF & ZDCF \\
\hline cnt vs. $\mathrm{H} \alpha$ & 79 & $14.94_{-13.81}^{15.66}$ & $0.19_{-0.14}^{0.14}$ \\
cnt vs. $\mathrm{H} \alpha$ (poor Zeiss data discarded) & 60 & $16.29_{-140}^{14.30}$ & $0.28_{-0.16}^{0.15}$ \\
cnt vs. $\mathrm{H} \beta$ & 110 & $20.61_{-183}^{54.33}$ & $0.31_{-0.09}^{0.09}$ \\
cnt vs. $\mathrm{H} \beta$ (only Mexico data) & 80 & $10.75_{-9.76}^{19.31}$ & $0.15_{-0.13}^{0.13}$ \\
cnt vs. $\mathrm{H} \beta$ (without Zeiss data) & 95 & $16.85_{-14.86}^{20.13}$ & $0.34_{-0.11}^{0.10}$ \\
\hline
\end{tabular}

Notes. Column (1): analyzed light-curves. Column (2): number of spectra. Column (3): lag calculated using the ZDCF method. Column (4): cross-correlation coefficient calculated using the ZDCF method.

contribution to the $\mathrm{H} \alpha$ and $\mathrm{H} \beta$ emission line fluxes (given in absolute units, see Table 8). To estimate the host-galaxy continuum contribution, we used the Arp 102B and NGC 4339 (E0) spectra observed on the same night under the same good weather conditions (see Sect. 2.4). To subtract the NGC 4339 galaxy spectrum from that of Arp 102B, we used $\mathrm{Mg} \mathrm{Ib}$ and found that the best fit (when $\mathrm{Mg} \mathrm{Ib}$ and $\mathrm{Na}$ ID are completely removed from the composite spectra) is obtained with $75 \pm 3 \%$ of the host-galaxy contribution to the total Arp 102B continuum at $\sim 5100 \AA$ in the considered spectrum. However, depending on the AGN activity phase, the host galaxy contribution to the continuum at $\sim 5100 \AA$ was between $\sim 60 \%$ and $\sim 80 \%$ in the monitored period.

Additonally, we estimated the narrow-line contributions to the total and line-segment fluxes of $\mathrm{H} \alpha$ and $\mathrm{H} \beta$ (see Table 9). Below we will point out the parts where these contributions are important. 

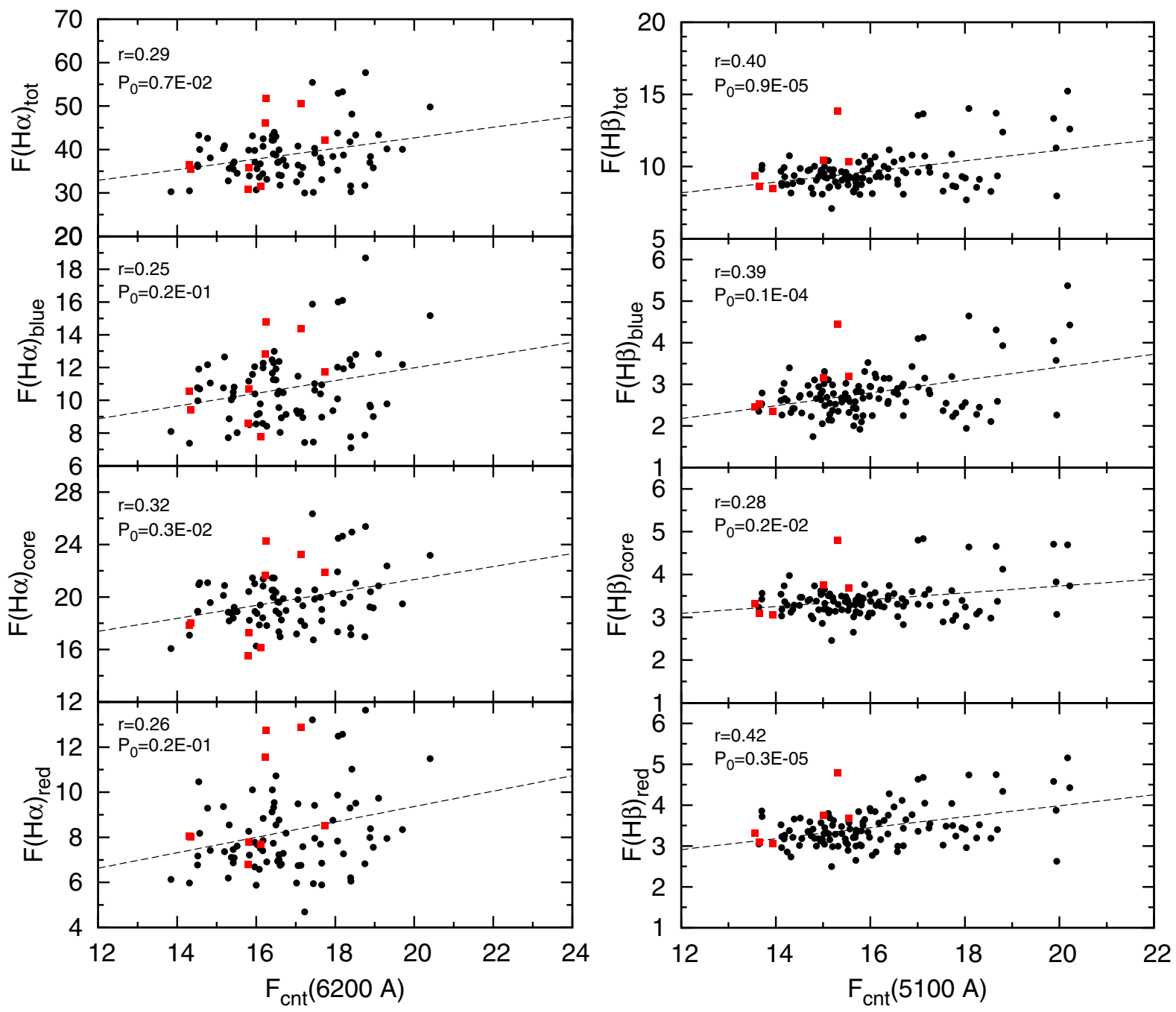

Fig. 8. $\mathrm{H} \alpha$ and $\mathrm{H} \beta$ line and their line-segment fluxes (blue wing, line core, red wing) vs. the continuum flux at 6300 and 5100 , respectively. The continuum flux is plotted in units of $10^{-16} \mathrm{erg} \mathrm{cm}^{-2} \mathrm{~s}^{-1} \mathrm{~A}^{-1}$, the line-segment fluxes in units of $10^{-14} \mathrm{erg} \mathrm{cm}^{-2} \mathrm{~s}^{-1}$. The correlation coefficient and the corresponding $p$-value are given in the upper left corner. The CA-data are denoted with squares.

\subsection{Variation of the broad lines and continuum}

As can be seen in Fig. 3, the broad line and continuum fluxes change by $10-30 \%$ during the monitored period (1987-2010). Some flares with amplitudes of $\sim 10-20 \%$ are observed, and these are especially strong in $\mathrm{H} \alpha$ and the continuum. The flarelike variation of Arp 102B was noted in Gezari et al. (2007).

The flux was observed to rise n 1987 (from Jun. 28 JD 2446975 to Jun. 30 - JD 2446977 ), and in 1998 (from May 6 - JD 2450940 to Jul. 26 - 2451021) in the $\mathrm{H} \alpha$ and $\mathrm{H} \beta$ emission lines of $30-40 \%$. It took the form of a flare-like event and lasted for about 80 days in 1998, but in the continuum such strong oserved flux changes were not observed. In Table 12 we show that the different observed mean line fluxes between 1987 and 1988-1994, and also between 1998 and 19992010 changed significantly, more than the continuum fluxes, by $32-35 \%$ in $\mathrm{H} \beta$ and $\sim 38-39 \%$ in $\mathrm{H} \alpha$, compared with $6-13 \%$ and $7-19 \%$ in the red and blue continuum, respectively.

However, after subtracting the host-galaxy contribution we found that in the same periods the relative variability amplitude of the mean corrected continuum (the AGNcontinuum) is significantly larger: $1987 /(1988-1994) \sim 1.3$ and $1998 /(1999-2010) \sim 1.6$ than it was in the hostgalaxy + AGN continuum: 1987/(1988-1994) 1.07 and
1998/(1999-2010) 1.19-1.13. These variations are comparable with the relative line flux variations.

A similar result was obtained for the parameter $F($ var $)-$ the variation amplitude of fluxes with respect to the mean flux (Table 10, host-galaxy-corrected data). As seen from Table 10, $F$ (var) in the corrected continuum fluxes (in the AGN-continuum) has a significantly larger continuum variability amplitude than the observed total continuum (for the continuum at $5100 \AA$, $31 \%$ against $~ 9 \%$ in the uncorrected spectra; for the continuum at $6200 \AA, 29 \%$ against $\sim 7 \%$ in the uncorrected spectra). On the other hand, $F$ (var) in the $\mathrm{H} \alpha$ and $\mathrm{H} \beta$ lines, remained almost unchanged after removing the contribution of the host-galaxy (see host-galaxy-corrected data in Table 10). In the corrected blue and red continuum there are some possible flares with an amplitude of up to $30 \%$ that last for a few (2-3) days (see Table 11), but these flares are not detected in the corrected $\mathrm{H} \alpha$ and $\mathrm{H} \beta$ line fluxes.

As noted above, we observed strong changes of the mean fluxes in the corrected continuum and almost constant changes of the mean flux in the broad lines, that is, a greatly reduced mean continuum flux by 1.3 times in 1988-1994 and 1.6 times in 1999-2010, while changes in the broad-line fluxes remained at the same level (1.3 times). The variability amplitude of the 

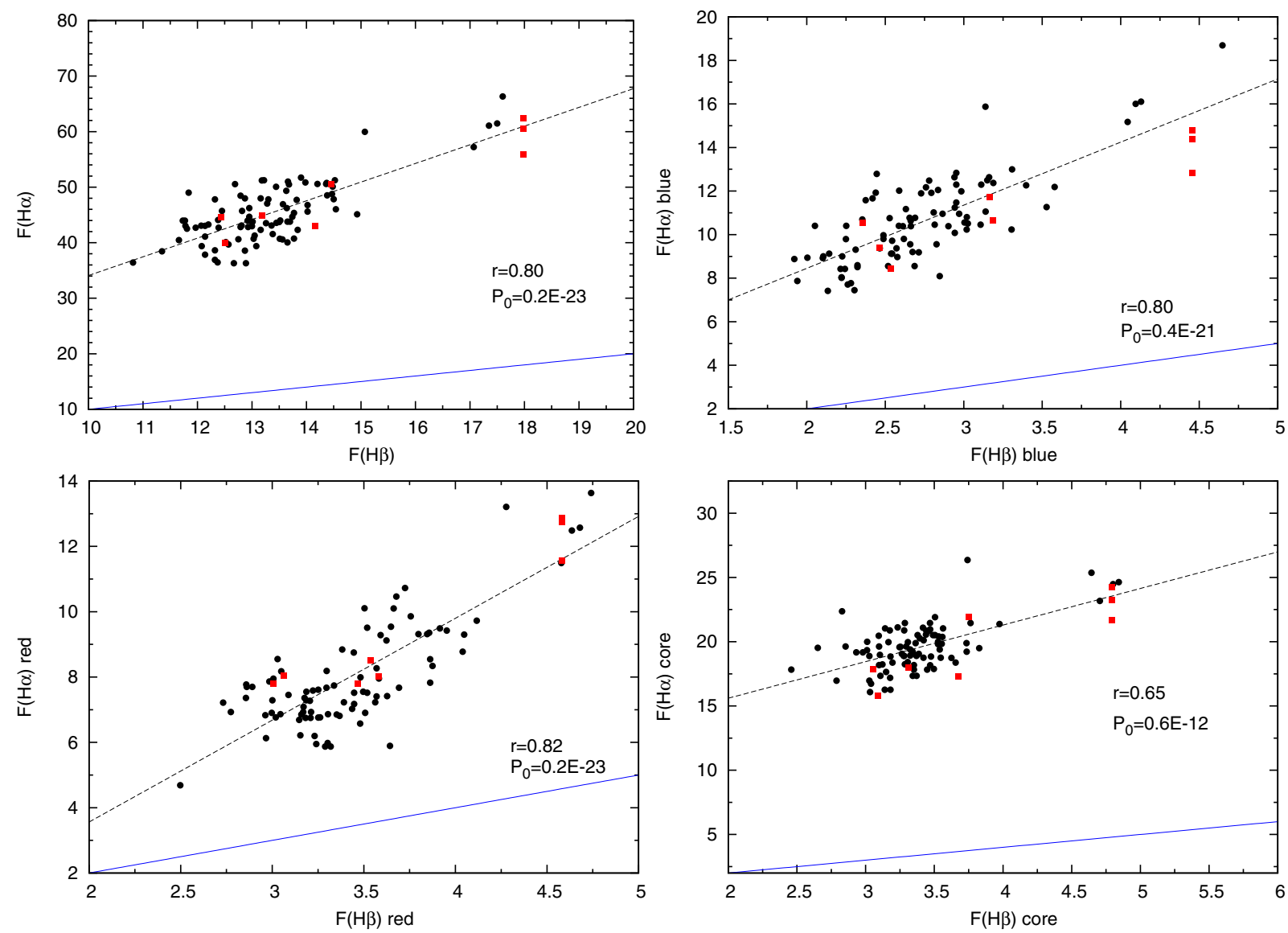

Fig. 9. $\mathrm{H} \alpha$ vs. $\mathrm{H} \beta$ total line and their line-segment fluxes (blue and red wing, line core). The correlation coefficient and the corresponding $p$-value are given in the bottom right corner. The CA-data are denoted with squares. The dashed line gives the linear best fit of the data, while the solid line marks the linear function with the slope equal to 1.

continuum and line fluxes remained more similar when we corrected the line fluxes for the narrow-line contributions, but a small correlation between the AGN-continuum and line fluxes remains (see Fig. 9).

Additionally, we note that a small amount of the optical continuum emission is probably only moderately effective in the broad-line reproduction. As for instance Chen \& Halpern (1989) have shown, the gravitational energy liberated in a standard accretion disk does not exceed the observed line emissivity in Arp 102B by much, therefore an additional source of heating may be required. These authors hypothesized that ion-supported tori might account for the unusual properties of Arp 102B. On the other hand, the variability amplitude of the optical continuum is similar to that of the line. Additionally, the variability amplitude and the intensity of the far-UV and X-ray continua is even strong enough to drive the line variability and intensity. It is also well-known (see e.g. Vagnetti, et al. 2013) that the variability of the high-energy bands (such as X-ray) is often more pronounced that the variability of the UV/optical band. But the question of the weak correlation between the continuum and line fluxes remains.

The most interesting result is the almost lacking correlation between the continuum and the broad lines $(r \sim 0.31$, see Figs. 4 and 5). We obtained the delays between the $\mathrm{H} \alpha$ and $\mathrm{H} \beta$ emission line fluxes and the continuum flux (lags $\sim 15-20$ days), see Table 13, using different CCF methods. However, the errors in the lag measurements are huge because the flux variability in the two lines and the continuum during the monitored period was small ( $\sim 10-20 \%$, see Table 10). It is difficult to calculate the lag for AGNs with very broad lines, (see e.g. NGC 7603 and Mrk 926 in Kollatschny et al. 2000; Kollatschny \& Zetzl 2010, respectively). The weak correlations between the line and continuum fluxes and the poor quality of the lag data also indicate a possible other source of photoionization in the BLR in addition to the central source (e.g. collisions of orbiting discrete clouds in the disk Sergeev et al. 2000).

Additionally, similar as some single-peaked AGNs (see e.g. Gilbert \& Peterson 2003), the $\mathrm{H} \beta$ and $\mathrm{H} \alpha$ lines show an intrinsic Baldwin effect, or a significant anticorrelation between the continuum flux and the equivalent widths of the broad lines (see Fig. 6). The anticorrelation tends to be stronger in $\mathrm{H} \beta$ (and is statistically more significant) than in the $\mathrm{H} \alpha$ line.

\subsection{Black hole mass in Arp $102 B$}

Most often, reverberation calculations have been applied to cases which give black hole masses within the range $10^{7}-10^{9} M_{\odot}$. At present, emission-line lags have been measured for a number of AGNs by using the cross-correlation between the continuum and emission-line light-curves.

Using the virial theorem, the mass of a black hole $\left(M_{\mathrm{BH}}\right)$ is (Peterson et al. 1998; Wandel et al. 1999)

$M_{\mathrm{BH}}=f \frac{\Delta V_{\mathrm{FWHM}}^{2} \cdot R_{\mathrm{BLR}}}{G}$, 

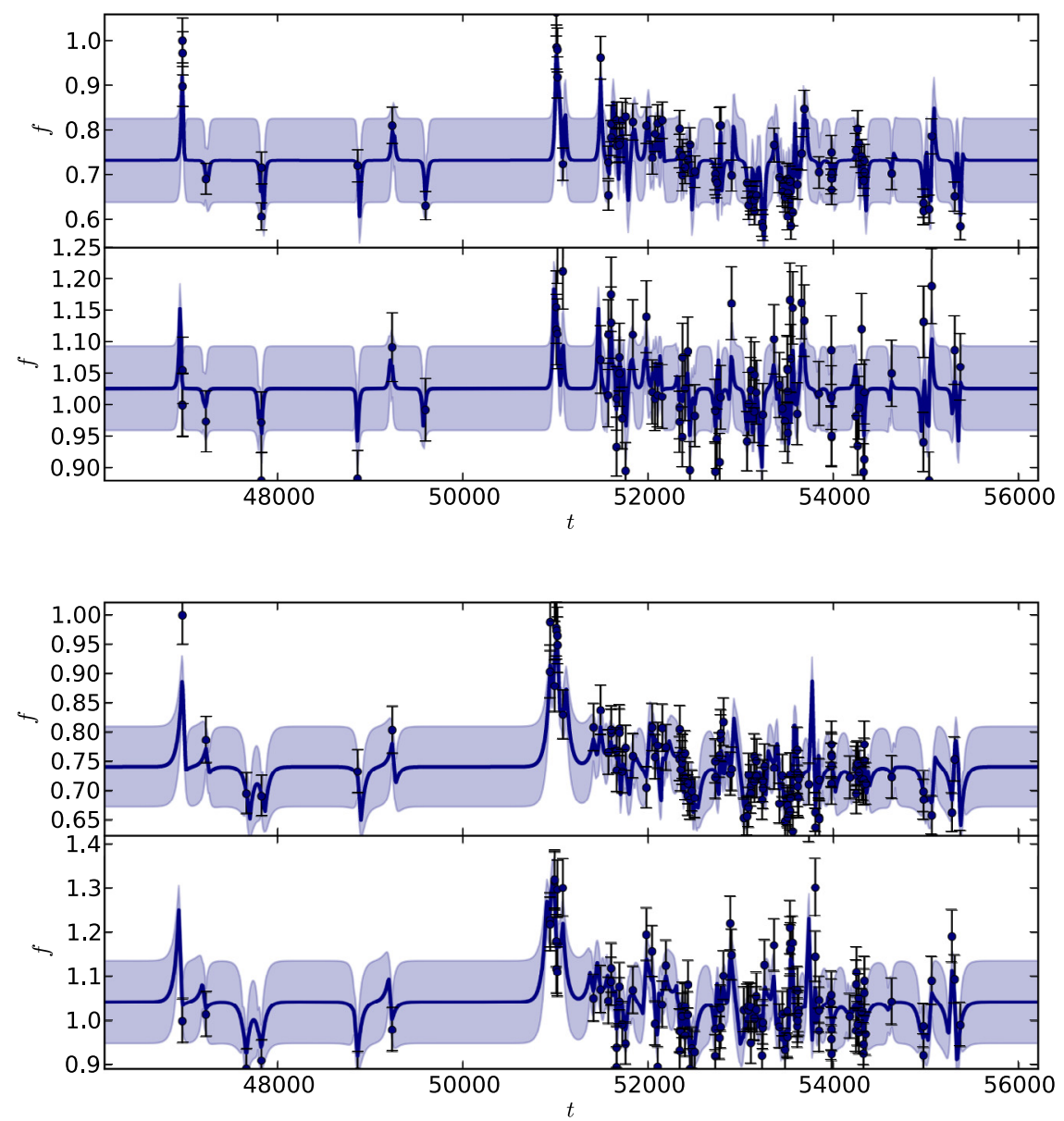

Fig. 10. Models of the $\mathrm{H} \alpha$ (upper panel) and $\mathrm{H} \beta$ line (lower panel), where the upper plot gives the line light-curve and the bottom plot the continuum lightcurve. In the plots the full line denotes the model of the expected mean light-curve, while dots represent the observed data. The blue band shows the expected spread of the light-curves around the mean consistent with the data. The $x$-axis gives the modified Julian Date (MJD), the $y$-axis the normalized line/continuum fluxes.
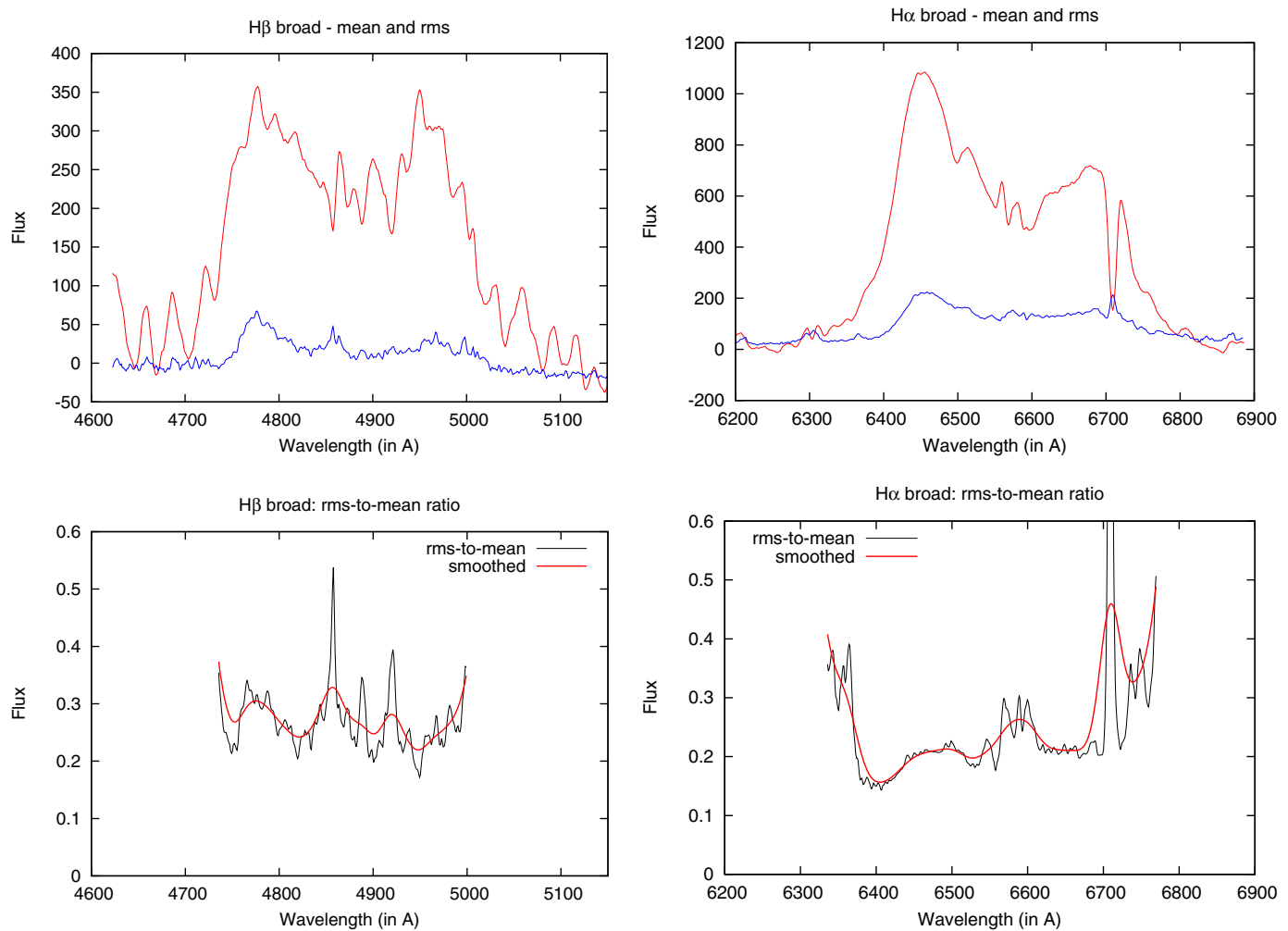

Fig. 11. Top panels give the mean and rms profiles, bottom panels the ratio of the rms-to-mean flux of the $\mathrm{H} \beta$ (left) and $\mathrm{H} \alpha($ right $)$ lines. 

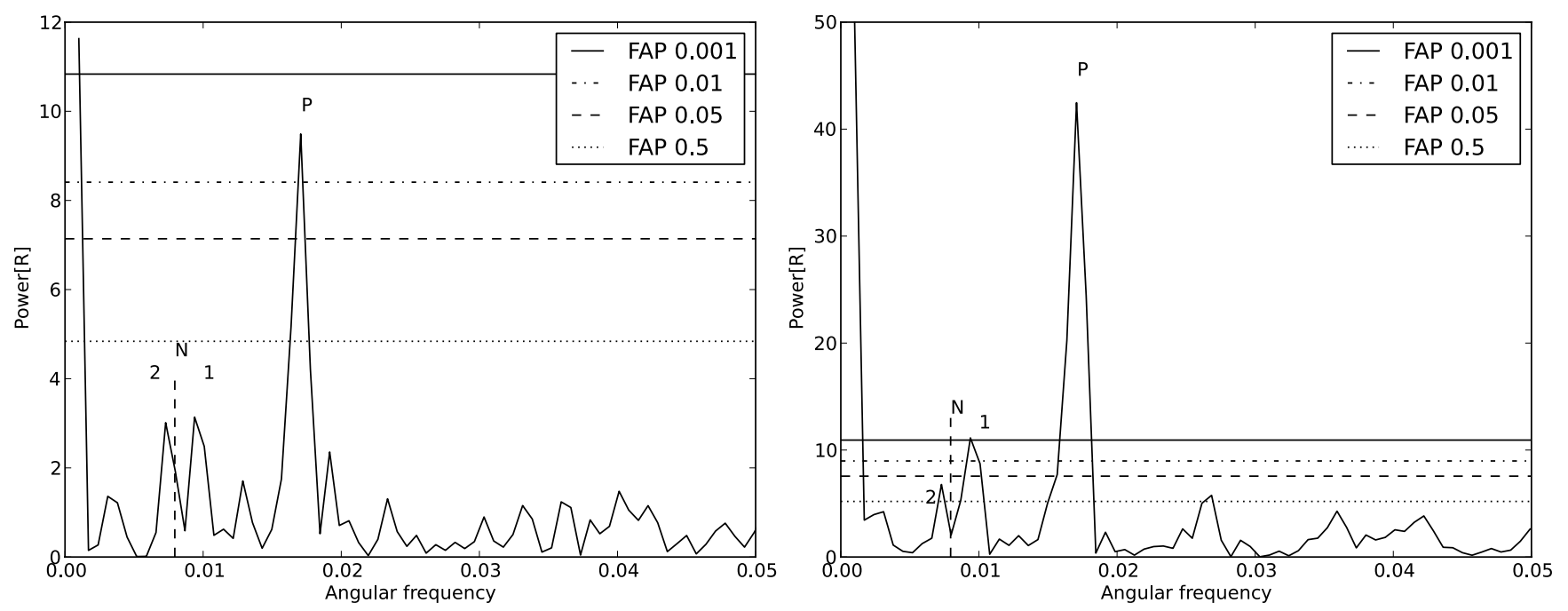

Fig. 12. Left: lomb-Scargle periodograms of the ratio of the red-to-blue line-segment fluxes $R$ of the $\mathrm{H} \alpha$ (left) and $\mathrm{H} \beta$ (right) lines (false-alarm probability lines are also indicated).

where $\Delta V_{\mathrm{FWHM}}$ is the orbital velocity at a radius $R_{\mathrm{BLR}}$ of the BLR, and it is estimated by the width of the emission line (specifically, the variable part of the line); $f$ is the factor that depends on the geometry of the BLR. Using a sample disk model for the Arp 102B BLR, one can use the relation derived in Onken et al. (2004)

$M_{\mathrm{BH}}=f \frac{\Delta V_{\mathrm{FWHM}}^{2} \cdot R_{\mathrm{BLR}}}{G} \cdot \frac{\sin (i)}{2 \ln (2)}$,

where $i$ is the inclination of the disk. Here we take $f=5.5$ as estimated by Onken et al. (2004) and $i \sim 30^{\circ}$ as given in Chen \& Halpern (1989).

The velocity dispersion of the disk can be estimated as (see La Mura et al. 2009)

$\Delta v=\frac{F W H M(\mathrm{H} \beta)}{8 \sin (i)}$,

where for our measurements $v_{\mathrm{FWHM}}(\mathrm{rms}) \approx 15000 \mathrm{~km} \mathrm{~s}^{-1}$ (Fig. 11b) and $i \sim 30^{\circ}$ gives $\Delta v=3750 \mathrm{~km} \mathrm{~s}^{-1}$. Taking into account that our estimate is $R_{\mathrm{BLR}} \approx 20$ light days (Table 13 ), we obtain a mass of the Arp 102B black hole of $\sim 1.1 \times 10^{8} M_{\odot}$.

There are other estimates of the BH mass in Arp 102B; using the hot-spot model to explain the line profile variations, Newman et al. (1997) estimated the black hole mass in Arp 102B to be $2.2 \times 10^{8} M_{\odot}$, while from the rotational clouds model, Sergeev et al. (2000) estimated the central body mass to be $3.5 \times 10^{8} M_{\odot}$.

It is interesting to compare the obtained BH masses mentioned above with the $M-\sigma *$ relation. Recently, the $M-\sigma *$ relation was given by Gültekin et al. (2009) as

$\log \left(M_{\mathrm{BH}} / M_{\odot}\right)=\alpha+\beta \log \left(\sigma * / 200 \mathrm{~km} \mathrm{~s}^{-1}\right)$,

where $\alpha=(8.12 \pm 0.08$ and $\beta=4.24 \pm 0.41$ are constants, and $\sigma *$ is the stellar disspersion. The disperssion velocity for Arp 102B was given by Barth et al. (2002) as $\sigma_{*}=188 \pm 8 \mathrm{~km} \mathrm{~s}^{-1}$ which results in an estimated Arp 102B black hole mass of $M_{\mathrm{BH}} \sim$ $1.01 \times 10^{8} M_{\odot}$.

Comparing the data obtained from our estimates and the estimates of Newman et al. (1997) and Sergeev et al. (2000), the $M-\sigma *$ relation gives a smaller mass for Arp 102B than estimated by Newman et al. (1997) and Sergeev et al. (2000) (two and three times, respectively). However, comparing our results and that obtained from the $M-\sigma_{*}$ relation, the agreement is very good $M_{\mathrm{rev}} / M_{M-\sigma *} \sim 1.1$.

The black hole masses of AGNs with double-peaked lines obtained with the $M-\sigma_{*}$ relation seem to disagree systematically with the virial masses obtained from one-epoch measurements (see Wu \& Liu 2004; Lewis \& Eracleous 2006). But it seems that the revereberation method gives better agreement, which is also true for another double-peaked line AGN, 3C 390.3, for which Dietrich et al. (2012) found that the reverberation-based mass of the $\mathrm{BH}$ also agrees with the $M-\sigma_{*}$ relations.

\section{3. $H \alpha$ and $H \beta$ flux variability}

We explored changes in the total line fluxes of $\mathrm{H} \alpha$ and $\mathrm{H} \beta$ and in the line segments (see Table 5). There are changes in the line profiles (blue and red double peaks, see Sect. 3.4) and their rms. The observed line parts in the two lines vary by about $20 \%$ (see Table 10). The correlations between the line segments are significant, but the slopes of the best fit are not consistent with one, only for the $\mathrm{H} \beta$ red wing vs. the $\mathrm{H} \beta$ line core does the slope follow 1 (see Fig. 7).

As seen from Fig. 8, there is only a weak correlation between fluxes of different segments of the two lines and the continuum flux $(r \sim 0.3-0.4)$. However, we found a very good correlation $(r \sim 0.8)$ between the $\mathrm{H} \alpha$ and $\mathrm{H} \beta$ fluxes (for the total line and for the line-segment fluxes, see Fig. 9), and between the fluxes of the blue, red, and core segments of the $\mathrm{H} \alpha$ and $\mathrm{H} \beta$ lines (Fig. 7); this agrees with the disk geometry.

On the other hand, we found an indication of periodical changes in the peak ratio (i.e., the red-to-blue line-segment flux ratio), with a period of about 370 days, which is shorter than the period obtained by Newman et al. (1997) and Gezari et al. (2007). However, we found two peaks in the periodogram very close to the period obtained by Newman et al. (1997) (with lower significance, especially for the $\mathrm{H} \alpha$ line, where the peaks are below $50 \%$ of the false-alarm probability line). Newman et al. (1997) pointed out that the determined period seems to be an orbital motion through the evolution of the ratio of red to blue fluxes (or, alternatively, through the evolution of parameter $\theta$, the azimuthal angular extent of the hot spot, in the models). Gezari et al. (2007) also interpreted the observed oscillation as 
two different bright spots orbiting at different radii in the disk at different times, but the authors mentioned a problem with this model, because the two bright spot rotation periods did not yield consistent values of the black hole mass. However, this scenario - two different bright spots orbiting at different radii successfully explains the line shape variability of 3C390.3 (see Jovanović et al. 2010).

Indeed, the only proposed source of variability in an AGN that would cause simple periodical sinusoidal variation in this ratio with little apparent decay (in amplitude or frequency) for nearly two complete cycles is orbital in nature. But the problem here is that there are indications for three possible rotation periods (two with lower significance close to the period given in Newman et al. 1997, and one that is almost twice as small).

Alternatively, it is possible that the rotation of the bright spot in the accretion disk is not purely Keplerian, e.g. the bright spot may be affected by a wave in the disk that rotates at a different speed (see Lewis et al. 2010). This may explain the multiperiodical oscillations seen in the spectra of Arp 102B, but we will consider this in more detail in the forthcoming paper.

\section{Conclusion}

We presented a long-term (1987-2010) spectroscopical observations of Arp 102B in the optical band. Arp 102B is an AGN with prominent double-peaked broad-line profiles. We investigated the continuum and line variations during this period, and from our investigations we can arrive at the following conclusions:

i) We found a significant contribution of the host-galaxy continuum (between $\sim 60 \%$ and $\sim 80 \%$ in the monitored period) to the total observed continuum in Arp 102B. The corrected (AGN) continuum flux of Arp 102b has an approximately flat shape and contributes about $\sim 25 \%$ to the total observed (host+AGN) continuum (Fig. 1, bottom). The flux variation of the AGN-continuum has a significantly larger amplitude than the observed total continuum (Table 10, $F($ var)). However, the $\mathrm{H} \alpha$ and $\mathrm{H} \beta$ line fluxes are not sensitive to the host-galaxy correction, that is, the variability amplitude remains almost unchanged (Table 10). As noted in Sect. 3, the corrected AGN-continuum shows some possible flare-like events, for instance, an increase in the flux by up to $>30 \%$ within a few (2-3) days (see Table 11), but corresponding flare-like events were not observed in the broadline $\mathrm{H} \alpha$ and $\mathrm{H} \beta$ light-curves.

ii) In different parts of the monitored periods, mean observed fluxes of the $\mathrm{H} \alpha, \mathrm{H} \beta$ broad emission lines and blue/red continuum variations are small (5-10\%, Table 10). But changes of the different mean fluxes in the lines between 1987 and (1988-1994), and between 1998 and (1999-2010) are significantly stronger than the continuum fluxes changes. However, after subtracting the host-galaxy contribution (in the same periods), the amplitude of the mean continuum flux changes (the AGN-continuum) is significantly larger $(1987 /(1988-1994) \sim 1.3$ and 1998/(1999-2010) 1.6). After removing the narrow-line contribution, the line flux amplitude variation, the relative AGN-continuum changes are similar to (or even stronger than) that of the flux changes in the lines. A rise in the $\mathrm{H} \alpha$ and $\mathrm{H} \beta$ emission line fluxes of about 30-40\% in 1987 and 1998 is similar to a long flare duration of about 80 days in 1998 (in 1987 observations are only 3 day long). However, the mean fluxes during the single years 1987, 1998 and during the periods 1988-1994 and
1999-2010 are constant within these periods within 5-10\% of the error bars (Table 12).

iii) The correlations between the observed fluxes of the $\mathrm{H} \alpha$ and $\mathrm{H} \beta$ lines and of their line segments with the continuum fluxes (Figs. 4-5) is very weak $(r \sim 0.3-0.4)$. This points to additional sources of ionization in the BLR apart from the central AGN continuum source. But there is a good linear relation between the observed fluxes in different line segments ( $r \sim 0.8$ ), that is, between the blue and red wings, and the line core (Fig. 7), and between the $\mathrm{H} \alpha$ and $\mathrm{H} \beta$ (Fig. 9) for the total line and line segment fluxes, which indicates the same geometry for the two emission regions. We also observed the anticorrelation between the continuum flux and equivalent widths of the $\mathrm{H} \alpha$ and $\mathrm{H} \beta$ lines (intrinsic Baldwin effect with $r \sim 0.37$ for $\mathrm{H} \alpha, r \sim 0.49$ for $\mathrm{H} \beta$, see Fig. 6), as in some Seyfert galaxies (Gilbert \& Peterson 2003).

iv) During the monitored period, the broad $\mathrm{H} \alpha$ and $\mathrm{H} \beta$ lines of Arp 102B showed double-peaked profiles. The blue peak is located around $-5000 \mathrm{~km} \mathrm{~s}^{-1}$, the red peak around $6000 \mathrm{~km} \mathrm{~s}^{-1}$ from the line center. The large distance between the peaks $\left(\sim 11000 \mathrm{~km} \mathrm{~s}^{-1}\right)$ indicates a fast rotating disk that is probably close to the black hole. As can be seen from Fig. 11 (mean and rms profiles), the changes in the line profile also have double-peaked rms, which indicates that the changes in the blue wing are significantly (some) stronger, than in the red wing. One central peak in the rms profile may be caused by a central component in the BLR (Popović et al. 2004; Bon et al. 2006, 2009).

v) From the Lomb-Scargle periodogram method that we applied on the measured red-to-blue peak flux ratio $(R=$ $F($ red $) / F$ (blue)) of $\mathrm{H} \alpha$ and $\mathrm{H} \beta$ lines, we found possible periodical variations (signals) in this ratio. We found a period of about 370 days and two additional peaks close to the period found by Newman et al. (1997).

vi) Several cross-correlation methods of the continuum and $\mathrm{H} \alpha$ and $\mathrm{H} \beta$ broad emission line fluxes indicates a lag of 15-20 days with large errorbars, caused by the weak flux variations in monitored period and the poor data sampling.

vii) From the mean and rms profiles of the two emission lines we found $F W H M(\mathrm{rms}) \sim 15000 \mathrm{~km} \mathrm{~s}^{-1}$ and from the CCF analysis we obtained a lag of $\sim 20$ days, thus we estimated a reverberation central black hole mass of $M_{\mathrm{rev}} \sim 1.11 \times 10^{8} M_{\odot}$, which is smaller than previous estimates (Newman et al. 1997; Sergeev et al. 2000), but agrees with the estimated black hole mass obtained from the $M-\sigma *$ relation.

The main property of the double-peaked Seyfert galaxy Arp 102B is the long-term variability on a timescale of months to some years, which is consistent with the dynamical timescale of an accretion disk. The observed long flare in $\mathrm{H} \alpha$ and $\mathrm{H} \beta$ with a duration of about 80 days in 1998 cannot be only due to the ionization by the AGNcontinuum source. Also, due to the lack of correlations with the AGN-continuum, this variability can be attributed to inhomogeneities in the line-emitting disk, such as hot spots, spiral arms, eccentricity, and warps. We will consider the nature of the broad spectral shape variability and consequently the BLR geometry in more detail in Paper II.

Acknowledgements. This work was supported by INTAS (grant N96-0328), RFBR (grants N97-02-17625, N00-02-16272, N03-02-17123, 06-02-16843, N09-02-01136, 12-02-00857a, 12-02-01237a), CONACYT research grants 39560, 54480, and 151494, PAPIIT-UNAM research grant IN111610 (Mexico), and the Ministry of Education and Science of Republic of Serbia through the project Astrophysical Spectroscopy of Extragalactic Objects (176001). L. Č.P., 
W.K., and D.I. are grateful to the Alexander von Humboldt foundation for support in the frame of the program "Research Group Linkage". W.K. is supported by DFG Project Ko 857/32-1. We thank Moiseev A. for providing some spectra of Arp 102B, obtained with the 6-m telescope. We would like to thank to the anonymous referee for very useful comments and suggestions.

\section{References}

Alexander, T. 2013 [arXiv: 1302 . 1508]

Antonucci, R., Hurt, T., \& Agol, E. 1996, ApJ, 456, L20

Barth, A. J., Ho, L. C., \& Sargent, W. L. 2002, AJ, 124, 2607

Bischoff, K., \& Kollatschny, W. 1999, A\&A, 345, 49

Bon, E., Popović, L. Č., Gavrilović, N., La Mura, G., \& Mediavilla, E. 2009, MNRAS, 400, 924

Bon, E., Popović, L., Ilić, D., \& Mediavilla, E. 2006, NewAR, 50, 716

Chen, K., \& Halpern, J. 1989, ApJ, 344, 115

Chen, K., Halpern, J. P., \& Filippenko, A. V. 1989, ApJ, 339, 742

Chen, K., Halpern, J. P., \& Titarchuk, L. G. 1997, ApJ, 483, 194

Dietrich, M., Peterson, B. M., Grier, C. J., et al. 2012, ApJ, 757, 53

Dimitrijević, M. S., Popović, L. Č., Kovačević, J., Dačić, M., \& Ilić, D. 2007, MNRAS, 374, 1181

Eracleous, M., \& Halpern, J. P. 1994, ApJS, 90, 1

Eracleous, M., Halpern, J., Gilbert, A., Newman, J. A., \& Filippenko, A. V. 1997, ApJ, 490, 216

Eracleous, M., Lewis, K. T., \& Flohic, H. M. L. G. 2009, NewAR, 53, 133

Fathi, K., Axon, D. J., Storchi-Bergmann, T., et al. 2011, ApJ, 736, 77

Flohic, H. M. L. G., \& Eracleous, M. 2008, ApJ, 686, 138

Gaskell, C. M. 2009, NewAR, 53, 140

Gilbert, K. M., \& Peterson, B. M. 2003, ApJ, 587, 123

Gezari, S., Halpern, J. P., Eracleous, M., \& Filippenko, A. V. 2004, IAUS, 222, 95

Gezari, S., Halpern, J. P., \& Eracleous, M. 2007, ApJS, 169, 167

Gültekin, K., Richstone, D. O., Gebhardt, K., et al. 2009, ApJ, 698, 198

Halpern, J. P., Eracleous, M., Filippenko, A. V., \& Chen, K. 1996, ApJ, 464, 704

Jovanović, P., Popović, L. Č., Stalevski, M., \& Shapovalova, A. I. 2010, ApJ, 718,168

Kollatschny, W., \& Zetzl, M. 2010, A\&A, 522, A36

Kollatschny, W., Bischoff, K., \& Dietrich, M., 2000, A\&A, 361, 901
La Mura, G., Di Mille, F., Ciroi, S., Popović, L. Č., \& Rafanelli, P. 2009, ApJ, 693, 1437

Lewis, K. T., \& Eracleous, M. 2006, ApJ, 642, 711

Lewis, K. T., Eracleous, M., \& Storchi-Bergmann, T. 2010, ApJS, 187, 416

Lomb, N. R. 1976, Ap\&SS, 39, 447

Miller, J. S., \& Peterson, B. M. 1990, ApJ, 361, 98

Newman, J. A., Eracleous, M., Filippenko, A. V., \& Halpern, J. 1997, ApJ, 485, 570

O’Brien, P. T., Dietrich, M., Leighly, K., et al. 1998, ApJ, 509, 163

Onken, C. A., Ferrarese, L., Merritt, D., Peterson, B. M., et al. 2004, ApJ, 615, 645

Peterson, B. M. 1993, PASP, 105, 207

Peterson, B. M., \& Collins II, G. W. 1983, ApJ, 270,71

Peterson, B. M., Berlind, P., Bertram, R., et al. 1994, ApJ, 425, 622

Peterson, B. M., Pogge, R. W., Wanders, I., Smith, S. M., \& Romanishin, W. 1995, PASP, 107, 579

Peterson, B. M., Wanders, I., Bertram, R., et al. 1998, ApJ, 501, 82

Peterson, B. M., Barth, A. J., Berlind, P., et al. 1999, ApJ, 510, 659

Peterson, B. M., Berlind, P., Bertram, R., et al. 2002, ApJ, 581, 197

Popović, L. Č., Mediavilla, E. G., \& Muñoz, J. A. 2001, A\&A, 378, 295

Popović, L. Č., Mediavilla, E., Bon, E., \& Ilić, D. 2004, A\&A, 423, 909

Popović, L. Č., Shapovalova, A. I., Ilić, D., et al. 2011, A\&A, 528, A130

Scargle, J. D. 1982, ApJ, 263, 835

Sergeev, S. G., Pronik, V. I., \& Sergeeva, E. A. 2000, A\&A, 356, 41

Shapovalova, A. I., Burenkov, A. N., Carrasco, L., et al. 2001, A\&A, 376, 775

Shapovalova, A. I., Doroshenko, V. T., Bochkarev, N. G., et al. 2004, A\&A, 422, 925

Shapovalova, A. I., Popović, L.Č., Collin, S., et al. 2008, A\&A, 486, 99

Shapovalova, A. I., Popović, L.C.., Bochkarev, N. G., et al. 2009, NewAR, 53, 191

Shapovalova, A. I., Popović, L.Č., Bochkarev, N. G., et al. 2010, A\&A, 517, A42 Shapovalova, A. I., Popović, L.Č., Burenkov, A. N., et al. 2012, ApJS, 202, 10 Stauffer, J., Schild, R., \& Keel, W. 1983, ApJ, 270, 465

Sulentic, J. W., Zheng, W., Calvani, M., \& Marziani, P. 1990, ApJ, 355, 15

Sulentic, J. W., Marziani, P., \& Dultzin-Hacyan, D. 2000, ARA\&A, 38, 521

Vagnetti, F., Antonucci, M., Trevese, D., \& Veron, P. 2013, A\&A, 550, A71

Van Groningen, E., \& Wanders, I. 1992, PASP, 104, 700

Wandel, A., Peterson, B. M., \& Malkan, M. A. 1999, ApJ, 526, 579

Wu, X.-B., \& Liu, F. K. 2004, ApJ, 614, 91

Zu, Y., Kochanek, C. S., \& Peterson, B. M. 2011, ApJ, 735, 80 